\title{
On the duration of the embedded phase of star formation
}

\author{
Jaeyeon Kim ${ }^{\odot},{ }^{1 \star}$ Mélanie Chevance ${ }^{\odot},{ }_{1}^{1}$ J. M. Diederik Kruijssen ${ }^{\oplus},{ }_{1}^{1}$ Andreas Schruba, ${ }^{2}$ \\ Karin Sandstrom, ${ }^{3}$ Ashley T. Barnes ${ }^{\oplus},{ }^{4}$ Frank Bigiel, ${ }^{4}$ Guillermo A. Blanc, ${ }^{5,6}$ Yixian Cao, ${ }^{7}$ \\ Daniel A. Dale, ${ }^{8}$ Christopher M. Faesi, ${ }^{9}$ Simon C. O. Glover ${ }^{\odot},{ }^{10}$ Kathryn Grasha ${ }^{\odot},{ }^{11}$ Brent Groves,${ }^{12}$ \\ Cinthya Herrera, ${ }^{13}$ Ralf S. Klessen ${ }^{\odot},{ }^{10,14}$ Kathryn Kreckel ${ }^{\oplus},{ }^{1}$ Janice C. Lee, ${ }^{15}$ Adam K. Leroy, ${ }^{16}$ \\ Jérôme Pety, ${ }^{13,17}$ Miguel Querejeta, ${ }^{18}$ Eva Schinnerer, ${ }^{19}$ Jiayi Sun ${ }^{\bullet},{ }^{16}$ Antonio Usero, ${ }^{18}$ Jacob L. Ward ${ }^{1}$ \\ and Thomas G. Williams ${ }^{\circledast 19}$
}

Affiliations are listed at the end of the paper

Accepted 2021 March 19. Received 2021 March 3; in original form 2020 November 30

\begin{abstract}
Feedback from massive stars plays a key role in molecular cloud evolution. After the onset of star formation, the young stellar population is exposed by photoionization, winds, supernovae, and radiation pressure from massive stars. Recent observations of nearby galaxies have provided the evolutionary timeline between molecular clouds and exposed young stars, but the duration of the embedded phase of massive star formation is still ill-constrained. We measure how long massive stellar populations remain embedded within their natal cloud, by applying a statistical method to six nearby galaxies at 20-100 pc resolution, using $\mathrm{CO}$, Spitzer $24 \mu \mathrm{m}$, and $\mathrm{H} \alpha$ emission as tracers of molecular clouds, embedded star formation, and exposed star formation, respectively. We find that the embedded phase (with CO and $24 \mu \mathrm{m}$ emission) lasts for 2-7 Myr and constitutes 17-47 per cent of the cloud lifetime. During approximately the first half of this phase, the region is invisible in $\mathrm{H} \alpha$, making it heavily obscured. For the second half of this phase, the region also emits in $\mathrm{H} \alpha$ and is partially exposed. Once the cloud has been dispersed by feedback, $24 \mu \mathrm{m}$ emission no longer traces ongoing star formation, but remains detectable for another 2-9 Myr through the emission from ambient CO-dark gas, tracing star formation that recently ended. The short duration of massive star formation suggests that pre-supernova feedback (photoionization and winds) is important in disrupting molecular clouds. The measured time-scales do not show significant correlations with environmental properties (e.g. metallicity). Future JWST observations will enable these measurements routinely across the nearby galaxy population.
\end{abstract}

Key words: stars: formation - ISM: clouds - galaxies: evolution-galaxies: ISM-galaxies: star formation.

\section{INTRODUCTION}

Massive stars $\left(>8 \mathrm{M}_{\odot}\right)$ form in the densest regions of molecular clouds. Once formed, these stars emit large quantities of ionizing photons creating HII regions and generate strong winds, which together alter the structure of their birth clouds and the surrounding interstellar medium. After a relatively short lifetime (4-20 Myr; Leitherer et al. 2014; Barnes et al. 2020; Chevance et al. 2020a), these massive stars die in supernova explosions injecting energy and momentum into their surroundings. Theoretical studies of giant molecular clouds (GMCs) indicate that these feedback processes are responsible for freeing the young stars from their parental clouds and destroying the GMCs (see e.g. Krumholz 2014; Dale 2015; Chevance et al. 2020b, for recent reviews). However, it is still debated which feedback mechanisms efficiently disrupt the birth clouds and which affect the diffuse interstellar medium on large scales (e.g. Lucas, Bonnell \& Dale 2020; Barnes et al. in preparation).

Observationally, several studies have constrained the time-scale for GMC destruction by stellar feedback in the Milky Way and in

* E-mail: kim@uni-heidelberg.de nearby galaxies using optical and ultraviolet (UV) star formation tracers, which are sensitive to recent, not heavily obscured star formation. Rapid dispersion of GMCs, within a cloud dynamical time-scale $(\leq 10 \mathrm{Myr})$, has been suggested based on the age distributions of stars in nearby star-forming regions and young stellar associations (Elmegreen 2000; Hartmann 2001). For GMCs in the Milky Way, M33, and the Large Magellanic Cloud (LMC), somewhat longer feedback time-scales of 10-20 Myr have been proposed by classifying molecular clouds into different types based on the (non)existence of their star formation activity (Engargiola et al. 2003; Blitz et al. 2007; Kawamura et al. 2009; Miura et al. 2012; Corbelli et al. 2017). Such methods have limitations because individual GMCs and star-forming regions need to be resolved. In addition, most of these studies only constrain the duration of the cloud dispersal after the young stars have become partially exposed. The exact role of each different feedback mechanism remains ambiguous, because the total duration of the embedded phase of star formation, including a heavily obscured phase, has not been quantified.

Studies of star-forming regions in the Milky Way and some nearby galaxies show that the embedded phase of massive star formation lasts for 2-5 Myr, where the duration of the heavily obscured phase is found to be $\sim 0.1-2 \mathrm{Myr}$ (Lada \& Lada 2003; Whitmore et al. 
2014; Corbelli et al. 2017). During the earliest stage of star formation, young massive stars are still embedded in their natal gas. As a result, $\mathrm{H} \alpha$ emission is heavily or partially obscured due to the extinction provided by the dust in dense gas surrounding the young stars. Despite this, on-going star formation is detectable using mid-infrared, hydrogen infrared recombination lines, radio recombination lines, and free-free radio continuum emission (Lockman 1989; Kennicutt 1998a; Calzetti et al. 2005; Kennicutt et al. 2007; Prescott et al. 2007; Murphy et al. 2011; Vutisalchavakul \& Evans 2013; Querejeta et al. 2019). In particular, the $24 \mu \mathrm{m}$ emission in the mid-infrared observed by the Multiband Imaging Photometer (MIPS) aboard the Spitzer Space Telescope provides an unbiased tracer of embedded massive star formation (e.g. see Kennicutt \& Evans 2012 for review), as long as the star-forming region has a fully sampled initial mass function (Vutisalchavakul \& Evans 2013). $24 \mu \mathrm{m}$ emission originates from stochastically heated small dust grains that do not require ionizing photons to be excited but do empirically correlate with tracers of massive star formation and so can be used as a tracer of the presence of large amounts of dust-reprocessed photospheric light from massive stars. Therefore, the $24 \mu \mathrm{m}$ emission is assumed to turn on only once massive stars are present. However, the radiation field from older stars (i.e. late-type B stars with an age of $\$ 100 \mathrm{Myr}$ ) can make a non-negligible contribution to the dust heating and thus the midinfrared dust emission (Draine \& Li 2007; Verley et al. 2009; Leroy et al. 2012). The exact contribution at $24 \mu \mathrm{m}$ wavelength is found to vary strongly between galaxies (e.g. 85 percent in M31 by Viaene et al. 2017, 20 percent in M33 by Williams et al. 2019, and up to around $>60$ per cent within the Galactic centre of the Milky Way, see Koepferl et al. 2015).

Following the theoretical model of star formation by Schmidt (1959), it is now observationally well-known that on galactic scales there is a tight correlation between the molecular gas surface density and the star formation rate (SFR) surface density (the 'star formation relation'; Silk 1997; Kennicutt 1998b; Bigiel et al. 2008; Leroy et al. 2013). However, this relation is also observed to break down on scales smaller than $\sim 1 \mathrm{kpc}$ (e.g. Onodera et al. 2010; Schruba et al. 2010; Ford et al. 2013; Leroy et al. 2013; Kreckel et al. 2018; Williams, Gear \& Smith 2018; Kruijssen et al. 2019; Schinnerer et al. 2019). This breakdown is caused by the small-scale de-correlation between GMCs and young stellar regions on sub-kpc scales and can be explained by assuming that individual regions in a galaxy follow independent lifecycles, during which clouds assemble, form stars, and get disrupted by feedback (Schruba et al. 2010; Feldmann, Gnedin \& Kravtsov 2011; Kruijssen \& Longmore 2014).

Kruijssen \& Longmore (2014) and Kruijssen et al. (2018) developed a formalism that translates the observed de-correlation quantitatively into the evolutionary timeline of GMCs from cloud formation to subsequent star formation, and finally cloud dispersal. In brief, this method uses the spatial variation of the gas-to-SFR flux ratio observed at different spatial scales ranging from cloud $(\sim 100 \mathrm{pc})$ to galactic $(\sim 1 \mathrm{kpc})$ scales for apertures placed on either gas peaks or SFR tracer peaks. These measurements are then used to determine the duration of each phase of the evolutionary cycle: the cloud lifetime, the time-scale a SFR tracer is visible, and the phase during which both molecular gas and SFR tracers overlap, which represents the duration of massive star formation as well as the timescale for molecular gas to be removed or dissociated by feedback. This method does not rely on high angular resolution as much as previous methods, using stellar ages or relative fractions of GMCs with and without internal star formation activity, since it only requires the mean separation length between regions undergoing independent evolution (100-200 pc; Chevance et al. 2020c) to be resolved instead of resolving individual star-forming regions (10-50 pc; Kawamura et al. 2009).

This method has been used to characterize the evolutionary timelines between molecular gas and exposed young stellar populations using $\mathrm{CO}$ and $\mathrm{H} \alpha$ observations. The first applications of this method to a number of galaxies covering a large range of galactic environments (Kruijssen et al. 2019; Chevance et al. 2020a,c; Hygate 2020; Ward et al. 2020a; Zabel et al. 2020) have shown that GMCs live for 10-30 Myr. CO and $\mathrm{H} \alpha$ emission are found to be coincident for 1-5 Myr, during which time feedback from the young stellar population disperses the molecular gas of their birth clouds. Considering that supernova explosions are expected 4-20 Myr after massive stars are formed (Leitherer et al. 2014; Chevance et al. 2020a), the short duration of overlapping $\mathrm{CO}$ and $\mathrm{H} \alpha$ emission suggests that pre-supernova feedback, such as photoionization and stellar winds, is important for disrupting star-forming molecular clouds (also see Barnes et al. 2020 Chevance et al. 2020a). Ward et al. (2020b) have also used this method and extended the evolutionary timeline of star-forming regions by incorporating $\mathrm{HI}$ emission to trace atomic gas. The measured atomic gas cloud lifetime in the LMC is $\sim 50 \mathrm{Myr}$ and almost no overlap with the exposed starforming phase is detected.

In this paper, we go a step further in the characterization of the GMC evolutionary lifecycle and use $\mathrm{CO}, 24 \mu \mathrm{m}$, and $\mathrm{H} \alpha$ emission as tracers for molecular gas, embedded star formation, and exposed star formation, respectively, for six nearby galaxies (IC 342, LMC, M31, M33, M51, and NGC 300). Previous applications of the statistical method to the same galaxies have focussed on characterizing the GMC lifecycle using $\mathrm{CO}$ emission as a tracer of the molecular gas and $\mathrm{H} \alpha$ emission as a tracer of the young massive stars (NGC 300: Kruijssen et al. 2019; M51: Chevance et al. 2020c; M33: Hygate 2020; and the LMC: Ward et al. 2020a). We derive novel measurements of the GMC lifetimes in IC 342 and M31 in this paper (see Appendix B); these are based on $\mathrm{CO}$ and $\mathrm{H} \alpha$ observations presented in Schruba et al. (2021a), Schruba et al. (2021b). Employing Spitzer $24 \mu \mathrm{m}$ observations allows us to probe the earliest phase of star formation where the stars are still heavily obscured, and quantify how long it takes young star-forming regions to emerge from their natal cloud (at which point the H II regions created by their ionizing radiation become visible).

The structure of this paper is as follows. In Section 2 and Appendix A, we describe the observational data used in our analysis. In Section 3, we summarize the statistical method used here and describe the associated input parameters for the galaxies in our sample. This is complemented by Appendix B, where we present in more detail the application of this method to IC 342 and M31. In Section 4, we then present the derived duration of the embedded massive star-forming phase, which can be separated into a heavily obscured phase of star formation and a partially exposed phase of star formation based on the existence of $\mathrm{H} \alpha$ emission. The total duration of the $24 \mu \mathrm{m}$ emitting phase is also presented. In addition, we explore how the durations of these phases vary with environmental properties, across the small galaxy sample for which these measurements are possible. We discuss the robustness of our results and compare them with the literature in Section 5. Last, we present our conclusions in Section 6.

\section{OBSERVATIONAL DATA}

We apply our analysis to six nearby galaxies: IC 342, the LMC, M31, M33, M51, and NGC 300. The size of our sample is mostly limited by the angular resolution of the Spitzer MIPS observations at $24 \mu \mathrm{m}$ 
( $\sim 6.4$ arcsec, Rieke et al. 2004; corresponding to 2-110 pc for the galaxies in our sample, including M51 for which we use a map at higher angular resolution, see Section 2.1) and the fact that we need to resolve at least the separation length between star-forming regions undergoing independent evolutionary lifecycles $(\sim 100-200 \mathrm{pc})$ to apply our analysis (see Section 5.1.1 and Kruijssen et al. 2018). Here, we provide a summary of the observational data used to trace the molecular gas (emission from low- $J$ CO transitions), embedded massive stars ( $24 \mu \mathrm{m}$ emission), and exposed young stellar regions ( $\mathrm{H} \alpha$ emission). Composite three-colour images of the $\mathrm{CO}, 24 \mu \mathrm{m}$, and $\mathrm{H} \alpha$ maps are presented in Fig. 1, whereas the individual $\mathrm{CO}$ and $24 \mu \mathrm{m}$ maps are shown in Appendix A. The physical and observational properties of the galaxies in our sample are listed in Table 1.

\subsection{Descriptions of multiwavelength data sets}

IC 342. We adopt a distance of $3.45 \mathrm{Mpc}$ (Wu et al. 2014), an inclination of $31.0^{\circ}$, and a position angle of $42.0^{\circ}$ (Meidt, Rand \& Merrifield 2009). The adopted metallicity at the galactic centre is $12+\log (\mathrm{O} / \mathrm{H})_{0}=8.83 \pm 0.04$, with a radial gradient of $-0.0500 \pm 0.0085 \mathrm{dex} \mathrm{kpc}^{-1}$, as measured by Pilyugin et al. (2014) using the strong-line 'counterpart' method by Pilyugin, Grebel \& Mattsson (2012). We use a combination of NOEMA and IRAM 30-m observations of the ${ }^{12} \mathrm{CO}(J=1-0)$ transition (denoted as $\mathrm{CO}(1-$ 0 ) in the following) from Schruba et al. (2021a) to trace molecular gas in IC 342 , covering out to $7.7 \mathrm{kpc}$ in galactic radius. The final data cube has a circular beam size of 3.6 arcsec, a spectral resolution of $5 \mathrm{~km} \mathrm{~s}^{-1}$, and a sensitivity of $135 \mathrm{mK}$ per $5 \mathrm{~km} \mathrm{~s}^{-1}$ velocity channel. Integrating over $10 \mathrm{~km} \mathrm{~s}^{-1}$, this translates to a $5 \sigma$ point source sensitivity of $5 \sigma\left(\mathrm{M}_{\mathrm{H}_{2}}\right) \approx 6.1 \times 10^{4} \mathrm{M}_{\odot}$ when the $\mathrm{CO}(1-0)$-to- $\mathrm{H}_{2}$ conversion factor $\left(\alpha_{\mathrm{CO}}\right)$ listed in Table 2 is assumed. In order to trace embedded star formation, we retrieved Spitzer MIPS $24 \mu \mathrm{m}$ image from the Local Volume Legacy (LVL) Survey (Dale et al. 2009). In addition to the procedure described in Dale et al. (2009), we mask foreground stars with a G-band magnitude $\leq 22$ mag using the Gaia DR2 catalogue (Gaia Collaboration 2018), as well as background galaxies via visual inspection of extended and non-circular sources. We then subtract background emission by fitting a plane determined at $2-2.5 R_{25}$ from the galactic centre where $R_{25}$ is the optical radius of the galaxy, obtained from the Lyon Extragalactic Database (Paturel et al. 2003a,b; Makarov et al. 2014). $\mathrm{H} \alpha$ emission has been observed with the Mosaic Imager on the Mayall 4-m telescope. We utilize calibrated narrow-band $\mathrm{H} \alpha$ and R-band images kindly provided by Kimberly Herrmann (private communication). We post-process these images as described in Schruba et al. (2021a). In brief, we subtract continuum emission from the $\mathrm{H} \alpha$ image, mask Milky Way stars using the Gaia DR2 catalogue, subtract a sky background by fitting a $1^{\text {st }}$ order polynomial plane at galactic radii $>10 \mathrm{kpc}$, and correct for Galactic extinction adopting $A(\mathrm{H} \alpha)=1.3$ mag which is derived from $\mathrm{E}(B-V)=0.494$ mag (Schlafly \& Finkbeiner 2011) and an extinction curve with $R_{\mathrm{V}}=3.1$ (Cardelli, Clayton \& Mathis 1989). ${ }^{1}$

\footnotetext{
${ }^{1}$ As explained in the discussion of each galaxy, we do not adopt the same literature for the reddening $(E(B-V))$ or the extinction law when correcting for Galactic extinction. This is to follow what has been done previously for each galaxy, in our first applications of the statistical method using $\mathrm{CO}$ and $\mathrm{H} \alpha$ emission only (Kruijssen et al. 2019; Chevance et al. 2020c; Hygate 2020; Ward et al. 2020a). Nevertheless, similarly to the $\mathrm{CO}(1-0)-$ to- $_{2}$ and SFR conversion factor (see Section 3.2), we note that our choice of correction factor does not affect our measurements of time-scales and region separation length, but only the derived total SFR and the integrated star formation efficiency.
}

For all the $\mathrm{H} \alpha$ maps used here, we do not attempt to correct for the internal extinction so that it traces exposed star-forming regions. The resulting $\mathrm{H} \alpha$ map has an angular resolution of 0.85 arcsec. Due to limited coverage of the $\mathrm{CO}$ survey and blending of bright sources at the galaxy centre, we restrict our analysis to regions where $\mathrm{CO}$ observations have been made within galactic radii $1.0-7.7 \mathrm{kpc}$, as shown in Fig. 1.

LMC. We adopt a distance of $50 \mathrm{kpc}$ (Pietrzyński et al. 2019), an inclination of $22.0^{\circ}$, and a position angle of $168.0^{\circ}$ (Kim et al. 1998). The adopted metallicity at the centre of the LMC is $12+\log (\mathrm{O} / \mathrm{H})_{0}=8.35 \pm 0.03$, with a radial gradient of $0.0105 \pm 0.0105 \mathrm{dex} \mathrm{kpc}^{-1}$, as measured by Toribio San Cipriano et al. (2017) using a direct measurement of electron temperature ( $\left.T_{\mathrm{e}}\right)$ from spectra of $\mathrm{H}$ II regions (direct $T_{\mathrm{e}}$-based method). We employ the $\mathrm{CO}(1-0)$ data presented in the third data release of the Magellanic Mopra Assessment (MAGMA; Wong et al. 2011, 2017) to trace molecular gas. MAGMA is a CO mapping survey of the LMC and SMC using the Mopra 22-m Telescope at the Australia Telescope National Facility. For the LMC, the observations were conducted as a follow-up study of the NANTEN survey (Fukui et al. 2008) by targeting a subset of previously identified molecular clouds ( $\sim 160$ out of 272 clouds), with an improved resolution in order to resolve the GMCs $(\sim 11 \mathrm{pc})$. In Fig. 1, white dotted lines show the coverage of the MAGMA survey and solid white circles indicate regions where molecular gas has been detected by the NANTEN survey, but not further targeted with the MAGMA survey. We exclude these white circled regions, where we know GMCs exist, from our analysis. ${ }^{2}$ However, we still include other regions not observed by the MAGMA survey, which might have diffuse and faint $\mathrm{CO}$ emission. We note that the inclusion of these sightlines, not covered by the MAGMA survey, should have a negligible impact on our results because diffuse and faint emission is eventually removed in our analysis through a filtering process (see Section 3). Moreover, the MAGMA survey covers most $(\sim 80$ percent $)$ of the total CO emission from the LMC observed by the NANTEN survey (Wong et al. 2011). The observed CO emission from the MAGMA survey also shows good agreement with the molecular gas map produced using dust continuum emission modelling (Jameson et al. 2016), indicating that we cover most of the emission from GMCs. The resulting angular resolution of the MAGMA CO data is 45 arcsec, and the sensitivity is $0.3 \mathrm{~K}$ per $0.526 \mathrm{~km} \mathrm{~s}^{-1}$ velocity channel. Integrating over $10 \mathrm{~km} \mathrm{~s}^{-1}$, this translates to a $5 \sigma$ point source sensitivity of $5 \sigma\left(\mathrm{M}_{\mathrm{H}_{2}}\right) \approx 2.2 \times 10^{3} \mathrm{M}_{\odot}$ (assuming the $\alpha_{\mathrm{CO}}$ from Table 2). The Spitzer MIPS $24 \mu \mathrm{m}$ image is from the SAGE project (Meixner et al. 2006), covering $7^{\circ} \times 7^{\circ}$ of the galaxy. The continuum subtracted $\mathrm{H} \alpha$ map is from the Southern H-Alpha Sky Survey Atlas (Gaustad et al. 2001) and has a resolution of 48 arcsec. We correct for Galactic extinction using $A(\mathrm{H} \alpha)=0.16$ mag, which is derived from $\mathrm{E}(\mathrm{B}-\mathrm{V})=0.06 \mathrm{mag}$ (Staveley-Smith et al. 2003) and an extinction curve with $R_{\mathrm{V}}=3.1$ (Cardelli et al. 1989). For the analysis here, we include emission from $0-3 \mathrm{kpc}$ in galactic radius, where the outer boundary is indicated in Fig. 1.

M31. We adopt a distance of $0.78 \mathrm{Mpc}$ (Dalcanton et al. 2012), an inclination of $37.7^{\circ}$, and a position angle of $77.7^{\circ}$ (Corbelli et al. 2010). The adopted metallicity at the galactic centre is $12+\log (\mathrm{O} / \mathrm{H})_{0}=8.8 \pm 0.1$, with a radial gradient of

\footnotetext{
${ }^{2}$ Using the cloud catalogue from the NANTEN survey (Fukui et al. 2008), Ward et al. (2020a) have modelled the CO emission from the GMCs that were not observed by MAGMA and have shown that excluding these regions from our analysis has a negligible effect on our measurements.
} 

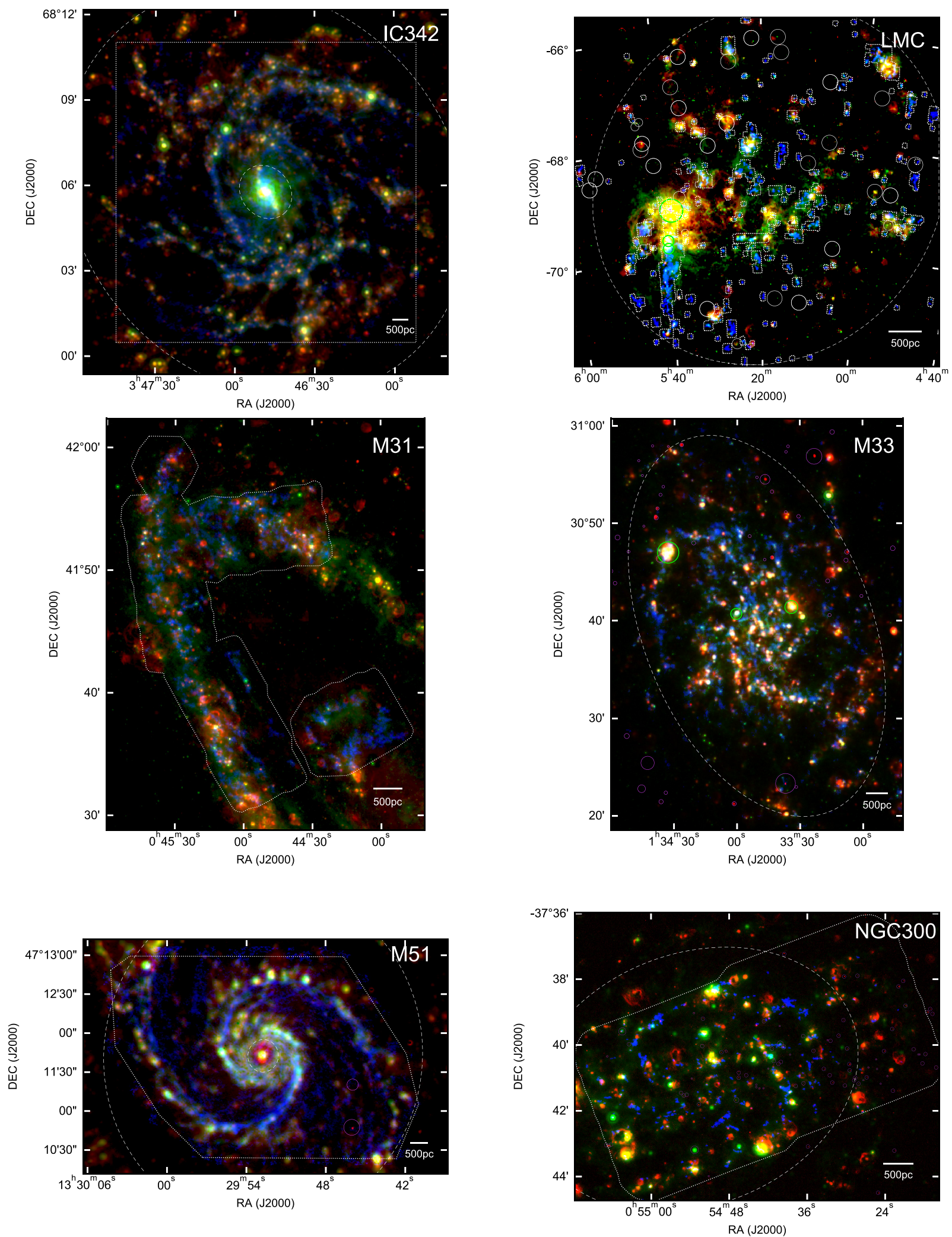

Figure 1. Composite three-colour images of the six galaxies in our sample. The $\mathrm{CO}(1-0)$ emission $(\mathrm{CO}(2-1)$ for M33) is presented in blue, Spitzer MIPS $24 \mu \mathrm{m}$ in green, and $\mathrm{H} \alpha$ in red. Galaxies are sorted by alphabetical order. The range of galactic radii included in the analysis is indicated by the white dashed ellipses. The inner ellipses of IC 342 and M51 show galaxy centres excluded from our analysis. The white dotted line shows the area where CO emission was observed in each galaxy (excluding the high noise edges of the map) and is not shown for M33 because it is outside the field of view. The massive star-forming regions of 30 Doradus in the LMC and NGC 604 in M33, as well as bright $24 \mu \mathrm{m}$ peaks are masked (green circles; see Section 2.3). In the LMC, regions that were not targeted by the MAGMA survey but are known to host gas clouds are masked (white solid circles). Foreground stars that were not removed in the image reduction process and image artefacts are also masked (purple circles). A physical scale of $500 \mathrm{pc}$ is shown in each image. 
Table 1. Physical and observational properties of our galaxy sample.

\begin{tabular}{|c|c|c|c|c|c|c|c|c|c|}
\hline Galaxy & $\begin{array}{l}\text { Stellar mass }^{a} \\
\left(\log _{10} \mathrm{M}_{\odot}\right)\end{array}$ & $\begin{array}{c}\text { Metallicity }{ }^{b, c} \\
\left(\mathrm{Z} / \mathrm{Z}_{\odot}\right)\end{array}$ & $\begin{array}{c}\text { Distance } \\
(\mathrm{Mpc})\end{array}$ & $\begin{array}{c}\text { Inclination } \\
\text { (deg) }\end{array}$ & $\begin{array}{c}\text { Position angle } \\
\text { (deg) }\end{array}$ & $\begin{array}{c}\text { CO } \\
\text { observations }\end{array}$ & $\begin{array}{c}\mathrm{CO} \\
\text { resolution } \\
(\operatorname{arcsec})\end{array}$ & $\begin{array}{l}24 \mu \mathrm{m} \\
\text { resolution } \\
(\operatorname{arcsec})\end{array}$ & $\begin{array}{l}\text { Spatial } \\
\text { resolution } \\
\quad(\mathrm{pc})\end{array}$ \\
\hline LMC & $9.3 \pm 0.1$ & $0.48 \pm 0.03$ & 0.05 & 22.0 & 168.0 & ATNF & 45 & 6.4 & 11 \\
\hline M31 (NGC 224) & $11.0 \pm 0.1$ & $0.76 \pm 0.20$ & 0.78 & 77.7 & 37.7 & $\begin{aligned} & \text { CARMA } \\
+ & \text { IRAM 30-m }\end{aligned}$ & 5.5 & 6.4 & 24 \\
\hline NGC 300 & $9.3 \pm 0.1$ & $0.48 \pm 0.06$ & 2.0 & 42.0 & 111.0 & ALMA & 2.1 & 6.4 & 62 \\
\hline
\end{tabular}

${ }^{a}$ Adopted from Skibba et al. (2012) for the LMC and Sick et al. (2015) for M31, while others are from Leroy et al. (2019).

${ }^{b} \mathrm{CO}$ luminosity weighted metallicity over the considered field of view.

${ }^{c}$ Obtained using $\mathrm{Z} / \mathrm{Z}_{\odot}=(\mathrm{O} / \mathrm{H}) /(\mathrm{O} / \mathrm{H})_{\odot}$, with the solar oxygen abundance $12+\log (\mathrm{O} / \mathrm{H})_{\odot}=8.69$ (Asplund et al. 2009).

${ }^{d}$ Coarsest spatial resolution of the $\mathrm{CO}$ and $24 \mu \mathrm{m}$ maps.

Table 2. Input parameters of the analysis using $24 \mu \mathrm{m}$ as SFR tracers for each galaxy. Other parameters not mentioned here are the same as in our previous analysis using $\mathrm{H} \alpha$ as an SFR tracer.

\begin{tabular}{|c|c|c|c|c|c|c|c|}
\hline Quantity & IC 342 & LMC & M31 & M33 & M51 & NGC 300 & Description \\
\hline$l_{\text {ap, } \max }(\mathrm{pc})$ & 3000 & 2000 & 3000 & 2500 & 3000 & 2560 & Maximum aperture size to convolve the input maps to \\
\hline $\mathrm{N}_{\mathrm{pix}, \min }$ & 20 & 10 & 20 & 20 & 10 & 100 & Minimum number of pixels for a valid peak \\
\hline$\delta \log _{10} \mathcal{F}_{\mathrm{CO}}$ & 0.05 & 0.15 & 0.02 & 0.10 & 0.05 & 0.10 & $\begin{array}{l}\text { Logarithmic interval between flux contour levels for molecular gas } \\
\text { peak } \\
\text { identification }\end{array}$ \\
\hline$\Delta \log _{10} \mathcal{F}_{24 \mu \mathrm{m}}$ & 3.8 & 2.8 & 2.3 & 3.0 & 4.0 & 2.0 & $\begin{array}{l}\text { Logarithmic range below flux maximum covered by flux contour } \\
\text { levels } \\
\text { for SFR tracer peak identification }\end{array}$ \\
\hline$t_{\text {ref, errmax }}(\mathrm{Myr})$ & 2.0 & 1.6 & 2.1 & 1.6 & 9.2 & 2.1 & Upwards uncertainty on reference time-scale \\
\hline $\operatorname{SFR}\left(\mathrm{M}_{\odot} \mathrm{yr}^{-1}\right)$ & 0.97 & 0.12 & 0.041 & 0.18 & 1.63 & 0.063 & Total SFR in the analysed area \\
\hline$\sigma(\mathrm{SFR})\left(\mathrm{M}_{\odot} \mathrm{yr}^{-1}\right)$ & 0.19 & 0.03 & 0.008 & 0.04 & 0.32 & 0.013 & Uncertainty of the total SFR \\
\hline $\log _{10} \alpha_{\mathrm{CO}}$ & 0.65 & 0.83 & 0.69 & 0.81 & 0.59 & 0.82 & Logarithm of $\mathrm{CO}(1-0)-$ to- $\mathrm{H}_{2}$ conversion factor \\
\hline$\sigma_{\text {rel }}\left(\alpha_{\mathrm{CO}}\right)$ & 0.5 & 0.5 & 0.5 & 0.5 & 0.5 & 0.5 & Relative uncertainty of $\alpha_{\mathrm{CO}}$ \\
\hline$n_{\lambda}$ & 13 & 7 & 10 & 10 & 16 & 8 & $\begin{array}{l}\text { Characteristic width for the Gaussian filter used to remove diffuse } \\
\text { emission in Fourier space }\end{array}$ \\
\hline
\end{tabular}

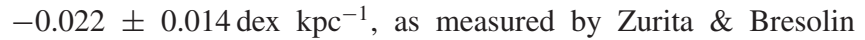
(2012) using the strong-line calibration from Pilyugin (2001). To trace molecular gas, we use $\mathrm{CO}(1-0)$ data first appeared in CaldúPrimo \& Schruba (2016), with full details presented in Schruba et al. (2021b). These data are obtained by combining CARMA interferometry data and IRAM 30-m data, the latter from Nieten et al. (2006). The CARMA observations cover $87 \mathrm{kpc}^{2}$ of M31's star-forming disc at galactic radii of $6-13 \mathrm{kpc}$. They have an angular resolution of 5.5 arcsec, a spectral resolution of $2.5 \mathrm{~km} \mathrm{~s}^{-1}$, and a sensitivity of $175 \mathrm{mK}$ per $2.5 \mathrm{~km} \mathrm{~s}^{-1}$ velocity channel. Integrating over $10 \mathrm{~km} \mathrm{~s}^{-1}$, this translates to a $5 \sigma$ point source sensitivity of $5 \sigma\left(\mathrm{M}_{\mathrm{H}_{2}}\right) \approx 7.3 \times 10^{3} \mathrm{M}_{\odot}$ (assuming the $\alpha_{\mathrm{CO}}$ from Table 2 ). We utilize the velocity masked moment-zero map, which is designed to be flux-complete (see Schruba et al. 2021b for details). We employ the Spitzer MIPS $24 \mu \mathrm{m}$ map presented in Gordon et al. (2006). This map is already background subtracted, and we refer the reader to the original paper for more information on the data reduction procedure. The $\mathrm{H} \alpha$ emission map is discussed in Schruba et al. (2021b) and has been created from calibrated narrow-band $\mathrm{H} \alpha$ and R-band images from the Local Group Galaxies Survey (Massey et al. 2006). The observations were carried out by the Mosaic Imager on the Mayall 4-m telescope. The calibrated data were post-processed as described in Schruba et al. (2021b), which include $\mathrm{H} \alpha$ continuum subtraction, masking of Milky Way stars using the Gaia DR2 catalogue (Gaia Collaboration 2018), a sky background subtraction by fitting a plane at galactic radii $>20 \mathrm{kpc}$, and a correction for the contamination by [N II] by assuming that both [N II] lines contribute 35 per cent of the total $\mathrm{H} \alpha$ emission, following Azimlu, Marciniak \& Barmby (2011). 
The Galactic extinction is corrected by adopting a factor $A(\mathrm{H} \alpha)=$ 0.14 mag, which is derived from $\mathrm{E}(\mathrm{B}-\mathrm{V})=0.05$ mag (Schlafly \& Finkbeiner 2011) and an extinction curve with $R_{\mathrm{V}}=3.1$ (Cardelli et al. 1989). The resulting $\mathrm{H} \alpha$ map has an angular resolutions of 1.5 arcsec. We perform our analysis on the field of view spanned by the CO observations.

M33. We adopt a distance of 0.84 Mpc (Gieren et al. 2013), an inclination of $55.08^{\circ}$, and a position angle of $201.1^{\circ}$ (Koch et al. 2018). The adopted metallicity at the galactic centre is $12+\log (\mathrm{O} / \mathrm{H})_{0}=$ $8.48 \pm 0.04$, with a radial gradient of $-0.042 \pm 0.010$ dex $\mathrm{kpc}^{-1}$, as measured by Bresolin (2011) using a direct $T_{\mathrm{e}}$-based method. We use the ${ }^{12} \mathrm{CO}(J=2-1)$ transition (denoted as $\mathrm{CO}(2-1)$ in the following) data presented in Gratier et al. (2010) and Druard et al. (2014) to trace molecular gas. The observations were carried out using the HEterodyne Receiver Array (Schuster et al. 2004) on the IRAM 30-m telescope covering the galaxy out to radii of $7 \mathrm{kpc}$. The resulting angular resolution is $12 \operatorname{arcsec}$ and the average noise level is $20 \mathrm{mK}$ per $2.6 \mathrm{~km} \mathrm{~s}^{-1}$ velocity channel. Integrating over $10 \mathrm{~km} \mathrm{~s}^{-1}$, this noise level translates to a $5 \sigma$ point source sensitivity of $5 \sigma\left(\mathrm{M}_{\mathrm{H}_{2}}\right) \approx 6.2 \times 10^{3} \mathrm{M}_{\odot}$ (assuming the $\alpha_{\mathrm{CO}}$ from Table 2). We retrieve Spitzer MIPS $24 \mu \mathrm{m}$ image from the LVL Survey (Dale et al. 2009). We then apply the same post-processing procedures as described above for IC 342. The narrow-band $\mathrm{H} \alpha$ data are from Greenawalt (1998). The observations were carried out using the Burrell-Schmidt 0.6-m telescope at the Kitt Peak National Observatory (KPNO). Detailed information about the image reduction process can be found in Hoopes \& Walterbos (2000). The Galactic extinction is corrected by using $A(\mathrm{H} \alpha)=0.1 \mathrm{mag}$, obtained from $\mathrm{E}(\mathrm{B}-\mathrm{V})=0.0413$ mag (Schlegel, Finkbeiner \& Davis 1998) and an extinction curve with $R_{\mathrm{V}}=3.1$ (Fitzpatrick \& Massa 2007). The resolution of the $\mathrm{H} \alpha$ emission map is 2.0 arcsec. We restrict our analysis to galactocentric radii $\leq 5 \mathrm{kpc}$, as outlined in Fig. 1.

M51. We adopt a distance of 8.6 Mpc (Jacobs et al. 2009), an inclination of $21.0^{\circ}$, and a position angle of $173.0^{\circ}$ (Colombo et al. 2014). The adopted metallicity at the galactic centre is $12+\log (\mathrm{O} / \mathrm{H})_{0}=$ $8.88 \pm 0.053$, with a radial gradient of $-0.0223 \pm 0.0037 \mathrm{dex} \mathrm{kpc}{ }^{-1}$, as measured by Pilyugin et al. (2014) using the strong-line 'counterpart' method (Pilyugin et al. 2012). We use the $\mathrm{CO}(1-0)$ data of the inner $10 \times 6 \mathrm{kpc}^{2}$ of the M51 presented in Pety et al. (2013) as part of the PdBI Arcsecond Whirlpool Survey (PAWS; Schinnerer et al. 2013). The surveyed region is visible in Fig. 1. The PdBI observations were carried out using A, B, C, and D configurations. The IRAM 30-m telescope was used to recover emission at low spatial frequencies. The final data have an angular resolution of 1.1 arcsec and a sensitivity of $0.39 \mathrm{~K}$ per $5 \mathrm{~km} \mathrm{~s}^{-1}$ velocity channel. Integrating over $10 \mathrm{~km} \mathrm{~s}^{-1}$, this noise level translates to a $5 \sigma$ point source sensitivity of $5 \sigma\left(\mathrm{M}_{\mathrm{H}_{2}}\right) \approx 8.9 \times 10^{4} \mathrm{M}_{\odot}$ (assuming the $\alpha_{\mathrm{CO}}$ from Table 2). The integrated intensity map was created by applying a mask to the data cube as described in Pety et al. (2013). Due to the limited resolution of Spitzer MIPS $24 \mu \mathrm{m}$ imaging (6.4 arcsec; Rieke et al. 2004), we can in principle only apply our method to galaxies closer than $\sim 5 \mathrm{Mpc}$. However, using the higher resolution (2.4 arcsec) $24 \mu \mathrm{m}$ map created by Dumas et al. (2011), we are able to expand the application of our method to M51, located at $8.6 \mathrm{Mpc}$. This map was created by applying the HiRes deconvolution algorithm (Backus et al. 2005) to the 5th Spitzer Infrared Nearby Galaxies Survey (SINGS; Kennicutt et al. 2003) data delivery (see Dumas et al. 2011 for more details). However, we note that the artefacts introduced by the deconvolution algorithm could potentially bias our analysis, especially for time-scale-related quantities, by modifying the distribution of the $24 \mu \mathrm{m}$ flux around bright peaks, limiting the interpretation of our results for this galaxy. The $\mathrm{H} \alpha$ emission map is also from SINGS (Kennicutt et al. 2003). The observations were carried out using the KPNO 2.1-m telescope with the CFIM imager. The map is corrected for Galactic extinction adopting a correction factor $A(\mathrm{H} \alpha)=0.08 \mathrm{mag}$, obtained from $E(B-V)=0.03 \mathrm{mag}$ (Schlafly \& Finkbeiner 2011) and an extinction curve with $R_{\mathrm{V}}=$ 3.1 (Fitzpatrick 1999).We also correct for the contamination by [N II] lines by scaling the map by a factor of 0.7 . The resolution of the $\mathrm{H} \alpha$ emission map is 1.83 arcsec. Because we lack $\mathrm{CO}$ observations of the outer galaxy and sources at the galaxy centre are affected by crowding and contamination from active galactic nucleus, we restrict our analysis to the field of view of the $\mathrm{CO}$ observations, and within galactic radii of $0.51-5.35 \mathrm{kpc}$, as indicated in Fig. 1 .

$N G C 300$. We adopt a distance of $2.0 \mathrm{Mpc}$ (Dalcanton et al. 2009), an inclination of $42.0^{\circ}$, and a position angle of $111.0^{\circ}$ (Westmeier, Braun \& Koribalski 2011). We adopt a metallicity of $12+\log (\mathrm{O} / \mathrm{H})_{0}=8.46 \pm 0.05$ at the galactic centre and a radial

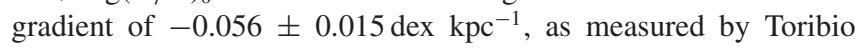
San Cipriano et al. (2016) using a direct $T_{\mathrm{e}}$-based method. We employ ALMA observations of the $\mathrm{CO}(1-0)$ transition, from ALMA programmes 2013.1.00351.S and 2015.1.00258.S (PI A. Schruba), to be presented in Schruba et al. (in preparation) and first used in Kruijssen et al. (2019). The observations were performed using the 12-m main array, as well as the 7-m array and total power antennas of the ALMA Compact Array, covering galactic radii out to $4.8 \mathrm{kpc}$. The resulting data have angular resolution of 2.1 $\operatorname{arcsec}(\sim 20 \mathrm{pc})$ and sensitivity of $0.1 \mathrm{~K}$ per $2 \mathrm{~km} \mathrm{~s}^{-1}$ channel. Integrating over $10 \mathrm{~km} \mathrm{~s}^{-1}$, this noise level translates to a $5 \sigma$ point source sensitivity of $5 \sigma\left(\mathrm{M}_{\mathrm{H}_{2}}\right) \approx 4.3 \times 10^{3} \mathrm{M}_{\odot}$ (assuming the $\alpha_{\mathrm{CO}}$ from Table 2). We retrieve Spitzer MIPS $24 \mu \mathrm{m}$ image from the LVL Survey (Dale et al. 2009) and apply the same postprocessing procedures as described above for IC 342. We use the $\mathrm{H} \alpha$ image presented in Faesi et al. (2014). This map is created from narrow-band $\mathrm{H} \alpha$ data and nearby continuum available in the ESO data archive, and we use here the version kindly shared by Chris Faesi (private communication). The observations were carried out with the Wide Field Imager on the MPG/ESO 2.2-m telescope at La Silla observatory. Correction for Galactic extinction is applied using $A(\mathrm{H} \alpha)=0.027 \mathrm{mag}$, obtained from $E(B-V)=0.01 \mathrm{mag}$ (Schlafly \& Finkbeiner 2011) and an extinction curve with $R_{\mathrm{V}}=$ 3.1 (Fitzpatrick 1999). We remove contamination of [N II] lines by assuming an intensity ratio $I(\mathrm{~N} I I) / I(\mathrm{H} \alpha)=0.2$. The resolution of the map is 1.35 arcsec. In our analysis, we consider emission from the field of view of the $\mathrm{CO}$ observations, and within $0-3 \mathrm{kpc}$ in galactic radius (beyond which the molecular gas surface density drops precipitously), and the outer boundary is visible in Fig. 1.

\subsection{Homogenization of maps to common pixel grid}

In order to apply our method, the gas and SFR tracer maps for a given galaxy need to share the same pixel grid. Therefore, for each galaxy, we regrid the map with a smaller pixel size to match the pixel grid of the map with larger pixel size. When the map that is being regridded has a better spatial resolution than the reference map, we first convolve the map with a Gaussian kernel to the resolution of the reference map before regridding to avoid introducing artifacts. ${ }^{3}$

\footnotetext{
${ }^{3}$ When convolving the $24 \mu \mathrm{m}$ map, we have also tested using a more exact kernel from Aniano et al. (2011) and found that the use of a Gaussian kernel has a negligible impact on our results.
} 


\subsection{Construction of masks}

We use the small-scale variation of the gas-to-SFR flux ratios to constrain the evolutionary timeline of the molecular clouds (see Section 3). By definition, our measurements are flux-weighted averages (see Kruijssen et al. 2018), which implies that very bright peaks dominating a significant fraction of the total flux can bias our results. Therefore, we mask star-forming regions in some galaxies that are clear outliers in the luminosity function of SFR tracer peaks. Specifically, we first sort the peak fluxes (identified using CLUMPFIND; see Section 3) in descending order. We then look for a gap in the distribution by calculating the ratio of the flux between the $n^{\text {th }}$ brightest and the next brightest peak in line, starting from the brightest peak. A gap is defined to exist when the $n^{\text {th }}$ peak is more than twice as bright as the $(n+1)^{\text {th }}$ peak. Whenever a gap is found, we mask all the peaks that are brighter than the $(n+1)^{\text {th }}$ brightest peak. As a result, we mask three star-forming regions each in the LMC and in M33 before applying our analysis (green circles in Fig. 1). These regions include 30 Doradus in the LMC and NGC 604 in M33, which alone contribute more than 30 per cent of the $24 \mu \mathrm{m}$ emission of each galaxy. Note that we would be masking the same peaks unless we go down to a brightness difference of 50 percent (rather than 100 percent) when defining a gap in the luminosity function. In this case, we would be masking one to four more peaks each in IC 342, M31, and NGC 300. The impact of masking such bright regions on the resulting derived parameters is generally small when averaging over the entire galaxy, but becomes significant if a smaller fraction of the galaxy is considered (see Ward et al. 2020a for the effect of 30 Doradus on the LMC and Chevance et al. 2020c for the effect of the 'headlight cloud' on the spiral galaxy NGC 628, also see Herrera et al. 2020). We also check for bright regions that satisfy this condition in the $\mathrm{CO}$ emission maps, but found none. Finally, we also mask artefacts in the maps (purple circles in Fig. 1).

\section{METHOD}

We employ a statistical method (formalized in the HEISENBERG code) to constrain the evolutionary timeline of GMCs. This timeline can be decomposed into the cloud lifetime, the duration of the embedded phase of star formation (which continues until dispersal of molecular clouds), and the star formation tracer lifetime. The characteristic separation length between star-forming regions undergoing independent evolution is also constrained in our analysis. Here, we provide a summary of the methodology and the main input parameters. We refer the reader to Kruijssen \& Longmore (2014) for a detailed explanation of the method, to Kruijssen et al. (2018) for the presentation and validation of the HEISENBERG code, as well as the full list of input parameters, and to Chevance et al. (2020c) for a general application of the method to nine nearby starforming galaxies. The accuracy of the method has been demonstrated in Kruijssen et al. (2018) using simulated galaxies, and has since been confirmed through extensive observational and numerical testing (Kruijssen et al. 2019; Haydon et al. 2020a; Ward et al. 2020b).

\subsection{Description of the analysis method}

Galaxies are composed of numerous GMCs and star-forming regions. The fundamental concept of our method is that such regions are independently undergoing their evolution, from molecular clouds to the formation of stars. These evolutionary phases are observed using gas (e.g. CO) and SFR tracers (e.g. $\mathrm{H} \alpha$ or $24 \mu \mathrm{m}$ ). We define the duration of each phase based on the visibility time-scale of the tracers used. The time-scale during which a gas emission tracer and an SFR tracer co-exist corresponds to the duration of massive star formation plus the time it takes to disrupt its natal molecular gas by stellar feedback (i.e. the feedback time-scale, $t_{\mathrm{fb}}$ ). In the following, the cloud lifetime will be denoted as $t_{\mathrm{CO}}$, the star formation tracer lifetime as $t_{\mathrm{H} \alpha}$ or $t_{24 \mu \mathrm{m}}$, and the feedback time-scale as $t_{\mathrm{fb}, \mathrm{H} \alpha}$, or $t_{\mathrm{fb}, 24 \mu \mathrm{m}}$ depending on the SFR tracer used.

During the initial phase of cloud evolution, a given independent region is only visible in the molecular gas tracer. As the cloud collapses and starts forming stars, the region becomes visible both in the gas and SFR tracers. Eventually, the remaining molecular gas is dispersed by stellar feedback and the region is only visible in the SFR tracers. Locally, the gas-to-SFR flux ratio therefore decreases with time during the evolution of a cloud. Observationally, when focussing on a non-star-forming GMC, a higher gas-to-SFR flux ratio is measured compared to the large-scale $(\sim 1 \mathrm{kpc})$ average gasto-SFR flux ratio. By contrast, when focussing on a young starforming region, where most of the molecular gas has been dispersed, a lower gas-to SFR flux ratio is measured. The deviations of the smallscale gas-to-SFR flux ratio compared to the large-scale average, as a function of spatial scale, can be directly related to the duration of the different phases of the GMC lifecycle (Kruijssen \& Longmore 2014; Kruijssen et al. 2018).

In practice, we first identify peaks in the gas tracer and SFR tracer emission maps. We then convolve both maps into a range of $N_{\text {ap }}$ spatial resolutions spanning from $l_{\text {ap, } \min }$ to $l_{\text {ap, } \max }$ (see Table 2). The minimum aperture size $\left(l_{\mathrm{ap}, \min }\right)$ is set to a value that is close to the size of the major axis of the deprojected beam of the coarsest resolution between the two maps, whereas the maximum aperture size $\left(l_{\mathrm{ap}, \max }\right)$ covers most of the galaxy. For each convolved map, apertures with the size of the corresponding resolution are placed on the identified gas and SFR tracer peaks. We then measure the gas and SFR tracer flux enclosed in these apertures to obtain the gas-to-SFR flux ratios as a function of aperture size. By fitting an analytical model describing the gas-to-SFR flux ratio as a function of the aperture size and the underlying evolutionary time-scales, we obtain a direct measurement of these time-scales. This can be understood with an idealized example. For a tracer that is longer lived, more peaks are typically identified, covering a larger fraction of the galaxy when small apertures are centred on them, compared to the shorter-lived tracer. The measured flux ratio is therefore closer to the galactic average value for a longer-lived tracer than a shorter-lived one.

We fit the analytical model derived by Kruijssen et al. (2018) to the measured flux ratios in order to constrain the relative duration of the different phases of the molecular cloud and star-forming region lifecycle, as well as the typical separation length between independent regions $(\lambda)$. The absolute duration of the different phases is then obtained by scaling the relative duration of timescales with a reference time-scale $\left(t_{\text {ref }}\right)$. In our previous analyses using $\mathrm{CO}$ and $\mathrm{H} \alpha$ observations (Kruijssen et al. 2019; Chevance et al. 2020c, a; Hygate 2020; Ward et al. 2020a), we used the duration of the isolated $\mathrm{H} \alpha$ emitting phase $\left(t_{\mathrm{ref}}=t_{\mathrm{H} \alpha}-t_{\mathrm{fb}, \mathrm{H} \alpha}\right)$, calibrated by Haydon et al. (2020b), Haydon et al. (2020a) using the stellar population synthesis model SLUG2 (da Silva, Fumagalli \& Krumholz 2012, 2014; Krumholz et al. 2015), as the reference timescale. Here, in order to obtain absolute values when applying our analysis to $\mathrm{CO}$ and $24 \mu \mathrm{m}$ maps, we first apply the method to $\mathrm{CO}$ and $\mathrm{H} \alpha$ observations. This is to obtain the cloud lifetime $\left(t_{\mathrm{CO}}\right)$ and its upward and downward uncertainties $\left(t_{\mathrm{CO}}\right.$, errmin and $t_{\mathrm{CO}}$, errmax ; see Table 3), which are adopted as the reference time-scale $\left(t_{\text {ref }}\right)$ and its uncertainties $\left(t_{\text {ref, errmin }}\right.$, and $\left.t_{\text {ref, errmax }}\right)$ in the analysis with $\mathrm{CO}$ 
Table 3. Physical quantities constrained using the method described in Section 3, describing the evolution of molecular clouds to exposed or embedded stellar populations traced by $\mathrm{H} \alpha$ and $24 \mu \mathrm{m}$, respectively. Following the notation throughout this paper, $t_{\mathrm{CO}}$ is the cloud lifetime, $t_{\mathrm{fb}, \mathrm{H} \alpha}$ and $t_{\mathrm{fb}, 24 \mu \mathrm{m}}$ are the duration of the partially exposed and embedded star-forming phase, respectively, and $t_{\mathrm{H} \alpha}$ and $t_{24} \mu \mathrm{m}$ are the duration of $\mathrm{H} \alpha$ and $24 \mu \mathrm{m}$ emitting phase, respectively. The region separation length $(\lambda)$ measured with different SFR tracers, feedback velocity $\left(v_{\mathrm{fb}}\right)$, and star-formation efficiency $\left(\epsilon_{\mathrm{sf}}\right)$ are also listed.

\begin{tabular}{|c|c|c|c|c|c|c|c|c|c|}
\hline \multirow[b]{2}{*}{ Galaxy } & \multirow[b]{2}{*}{$\begin{array}{c}t_{\mathrm{CO}} \\
(\mathrm{Myr})\end{array}$} & \multicolumn{3}{|c|}{$\mathrm{CO}$ versus $\mathrm{H} \alpha$} & \multicolumn{3}{|c|}{$\mathrm{CO}$ versus $24 \mu \mathrm{m}$} & \multirow[b]{2}{*}{$\begin{array}{c}v_{\mathrm{fb}} \\
\left(\mathrm{km} \mathrm{s}^{-1}\right]\end{array}$} & \multirow[b]{2}{*}{$\begin{array}{c}\epsilon_{\mathrm{sf}} \\
\text { (per cent) }\end{array}$} \\
\hline & & $\begin{array}{l}t_{\mathrm{fb}, \mathrm{H} \alpha} \\
(\mathrm{Myr})\end{array}$ & $\begin{array}{c}t_{\mathrm{H} \alpha} \\
(\mathrm{Myr})\end{array}$ & $\begin{array}{c}\lambda \\
(\mathrm{pc})\end{array}$ & $\begin{array}{c}t_{\mathrm{fb}, 24 \mu \mathrm{m}} \\
(\mathrm{Myr})\end{array}$ & $\begin{array}{l}t_{24 \mu \mathrm{m}} \\
(\mathrm{Myr})\end{array}$ & $\begin{array}{c}\lambda \\
(\mathrm{pc})\end{array}$ & & \\
\hline IC 342 & $20.0_{-2.3}^{+2.0}$ & $2.2_{-0.5}^{+0.4}$ & $6.4_{-0.6}^{+0.5}$ & $120_{-10}^{+10}$ & $5.2_{-2.3}^{+1.5}$ & $7.9_{-2.2}^{+1.8}$ & $190_{-62}^{+59}$ & $14.3_{-1.8}^{+4.0}$ & $1.9_{-0.8}^{+1.4}$ \\
\hline LMC & $11.1_{-1.7}^{+1.6}$ & $1.2_{-0.2}^{+0.2}$ & $5.8_{-0.4}^{+0.4}$ & $71_{-8}^{+13}$ & $5.0_{-2.0}^{+1.6}$ & $13.6_{-4.8}^{+3.7}$ & $73_{-26}^{+38}$ & $10.0_{-1.7}^{+2.1}$ & $6.8_{-3.0}^{+4.9}$ \\
\hline M31 & $14.0_{-1.9}^{+2.1}$ & $1.1_{-0.2}^{+0.3}$ & $5.5_{-0.3}^{+0.4}$ & $181_{-19}^{+28}$ & $2.4_{-0.8}^{+1.4}$ & $4.2_{-0.7}^{+1.5}$ & $128_{-23}^{+97}$ & $29.5_{-5.3}^{+6.9}$ & $0.7_{-0.2}^{+0.2}$ \\
\hline M33 & $14.5_{-1.5}^{+1.6}$ & $3.3_{-0.5}^{+0.6}$ & $7.9_{-0.6}^{+0.7}$ & $155_{-24}^{+30}$ & $6.8_{-2.0}^{+2.1}$ & $11.9_{-2.1}^{+2.9}$ & $119_{-35}^{+60}$ & $10.3_{-1.3}^{+1.5}$ & $3.5_{-1.5}^{+2.5}$ \\
\hline M51 & $30.7_{-4.9}^{+8.7}$ & $4.7_{-1.1}^{+2.0}$ & $8.9_{-1.2}^{+2.0}$ & $140_{-17}^{+25}$ & $<4.0^{a}$ & $3.6_{-0.9}^{+1.2}$ & $<136^{a}$ & $7.9_{-2.1}^{+2.0}$ & $3.3_{-1.4}^{+2.9}$ \\
\hline NGC 300 & $10.8_{-1.6}^{+2.2}$ & $1.5_{-0.2}^{+0.2}$ & $6.1_{-0.2}^{+0.2}$ & $104_{-18}^{+22}$ & $4.9_{-1.9}^{+1.2}$ & $7.9_{-2.1}^{+1.5}$ & $178_{-75}^{+125}$ & $9.4_{-0.7}^{+0.8}$ & $3.3_{-1.4}^{+2.6}$ \\
\hline
\end{tabular}

${ }^{a}$ Only a $1 \sigma$ upper limit can be derived for not satisfying (ii) and (viii) in Section 5.1.1.

and $24 \mu \mathrm{m}$ observations. The fitted model is thus described by three independent and non-degenerate quantities $\left(t_{24 \mu \mathrm{m}}, t_{\mathrm{fb}, 24 \mu \mathrm{m}}\right.$, and $\left.\lambda\right)$. The best-fitting values are then obtained by minimizing the reduced$\chi^{2}$ over these three quantities. The uncertainties of each parameter are propagated consistently throughout the analysis.

The presence of diffuse emission can bias our measurements by adding a large-scale component that is not associated with the identified peaks. This large-scale emission potentially includes diffuse emission originating from sources related to the recent massive star formation, such as low mass molecular clouds, low luminosity $\mathrm{H}$ II regions, and ionizing photons that have escaped from H II regions (e.g. Wood et al. 2010; Belfiore et al. in preparation). It may also originate from other mechanisms not related to recent massive star formation, for example, diffuse molecular gas, infrared emission powered by stars of intermediate age, and diffuse ionized gas created by shocks (Martin 1997; Leroy et al. 2012). We remove such diffuse emission in both gas and SFR tracer emission maps iteratively, using the method presented in Hygate et al. (2019), which makes the derived time-scales sensitive to only the massive/luminous molecular clouds and young stellar populations. This method filters emission on spatial scales larger than $n_{\lambda}$ times the typical distance between regions $\lambda$ (as measured from the HEISENBERG code) using a Gaussian high-pass filter in Fourier space. For each galaxy, we adopt the smallest possible value for $n_{\lambda}$, while ensuring the flux loss from the compact emission to be less than 10 percent (also following Chevance et al. 2020c; Hygate 2020; see Table 2). We do not adopt a fixed filtering scale because we want to maximize the removal of diffuse emission, while minimizing the impact of the filtering on the compact regions. The influence of $n_{\lambda}$ on the derived time-scales is fully described in Hygate et al. (2019) and Hygate (2020). In summary, the choice of $n_{\lambda}$ does not significantly change the best-fitting model parameters, as long as the adopted $n_{\lambda}$ is smaller than 30 and the flux loss from the compact emission is less than 10 percent. After the diffuse emission is filtered out, a noise mask with a threshold at twice the standard deviation noise level of the emission map is applied. We repeat this process until the convergence condition is reached, which is when the change of the measured value of $\lambda$ is less than 5 percent over three consecutive iterations.

\subsection{Input parameters}

Unless otherwise noted here, we adopt the same parameters as for previous analyses using $\mathrm{H} \alpha$ as an SFR tracer (see Appendix B for
IC 342 and M31, Ward et al. 2020a for the LMC, Hygate 2020 for M33, Chevance et al. 2020c for M51, and Kruijssen et al. 2019 for NGC 300). The parameters not mentioned here include distance, inclination, position angle (see Table 1), as well as parameters related to the fitting process and error propagation, for which default values are adopted as listed in Kruijssen et al. (2018). We use CLUMPFIND (Williams, de Geus \& Blitz 1994) to identify gas and SFR tracer peaks in each map. This algorithm finds peaks by drawing closed contours for a set of flux levels, within a given flux range $\left(\Delta \log _{10} \mathcal{F}\right)$ below the maximum flux level, with an interval of $\delta \log _{10} \mathcal{F}$ between flux levels. The adopted values for our sample are summarized in Table 2. Moreover, to avoid identifying point sources that are likely to be foreground stars that were not masked during the image reduction process or externally illuminated starless dust clumps (see Section 5.3), we only accept peaks that contain more than $N_{\text {pix, min }}$ pixels. The area of $N_{\text {pix, min }}$ pixels equals $0.2-3.5$ times the coarsest beam size. We note that our choices of $\Delta \log _{10} \mathcal{F}, \delta \log _{10} \mathcal{F}, N_{\text {pix }}$ min, $l_{\text {ap, max }}$, and $N_{\text {ap }}$ don't affect our measurements significantly as long as peaks that are obviously visible in the emission maps have been identified (Kruijssen et al. 2018). As explained above, $t_{\mathrm{CO}}$ and its uncertainties determined from our analysis with $\mathrm{CO}$ and $\mathrm{H} \alpha$ are used to define the reference time-scales $\left(t_{\text {ref }}\right)$. However, since we additionally mask some of the bright star-forming regions for the reasons explained in Section 2.3, we re-run the same analysis using $\mathrm{H} \alpha$ as an SFR tracer with updated masks. Our measurements using $\mathrm{H} \alpha$ as an SFR tracer are listed in Table 3, and are in very good agreement with (or identical to) the previously published results. For the analysis with $\mathrm{CO}$ and $24 \mu \mathrm{m}, t_{\text {ref }}\left(=t_{\mathrm{CO}}\right)$ includes the feedback phase. The $\mathrm{CO}(1-0)$-to- $\mathrm{H}_{2}$ conversion factor $\left(\alpha_{\mathrm{CO}}\right.$; including the contribution from heavy elements) is adopted from Bolatto, Wolfire \& Leroy (2013), expressed as

$\alpha_{\mathrm{CO}}=\left[2.9 \mathrm{M}_{\odot}\left(\mathrm{K} \mathrm{km} \mathrm{s}^{-1} \mathrm{pc}^{2}\right)^{-1}\right] \times \exp \left(\frac{0.4 \mathrm{Z}_{\odot}}{Z}\right)$.

We adopt the metallicity-dependent part, but not the surface density dependence from Bolatto et al. (2013). For simplicity, we adopt a constant $\alpha_{\mathrm{CO}}$ value for each galaxy and a conservative uncertainty of 50 per cent. In addition, for $\mathrm{M} 33$, which is the only galaxy with $\mathrm{CO}(2-$ 1) data, we adopt a fixed ratio of $\mathrm{CO}(2-1) / \mathrm{CO}(1-0)=0.8$ (Gratier et al. 2010). Finally, we derive the total SFR for the analysed area by combining $24 \mu \mathrm{m}$ and $\mathrm{H} \alpha$ emission maps and using the conversion factor from Calzetti et al. (2007), expressed as

$\operatorname{SFR}\left(\mathrm{M}_{\odot} \mathrm{yr}^{-1}\right)=5.3 \times 10^{-42}[L(\mathrm{H} \alpha)+0.031 L(24 \mu \mathrm{m})]$, 


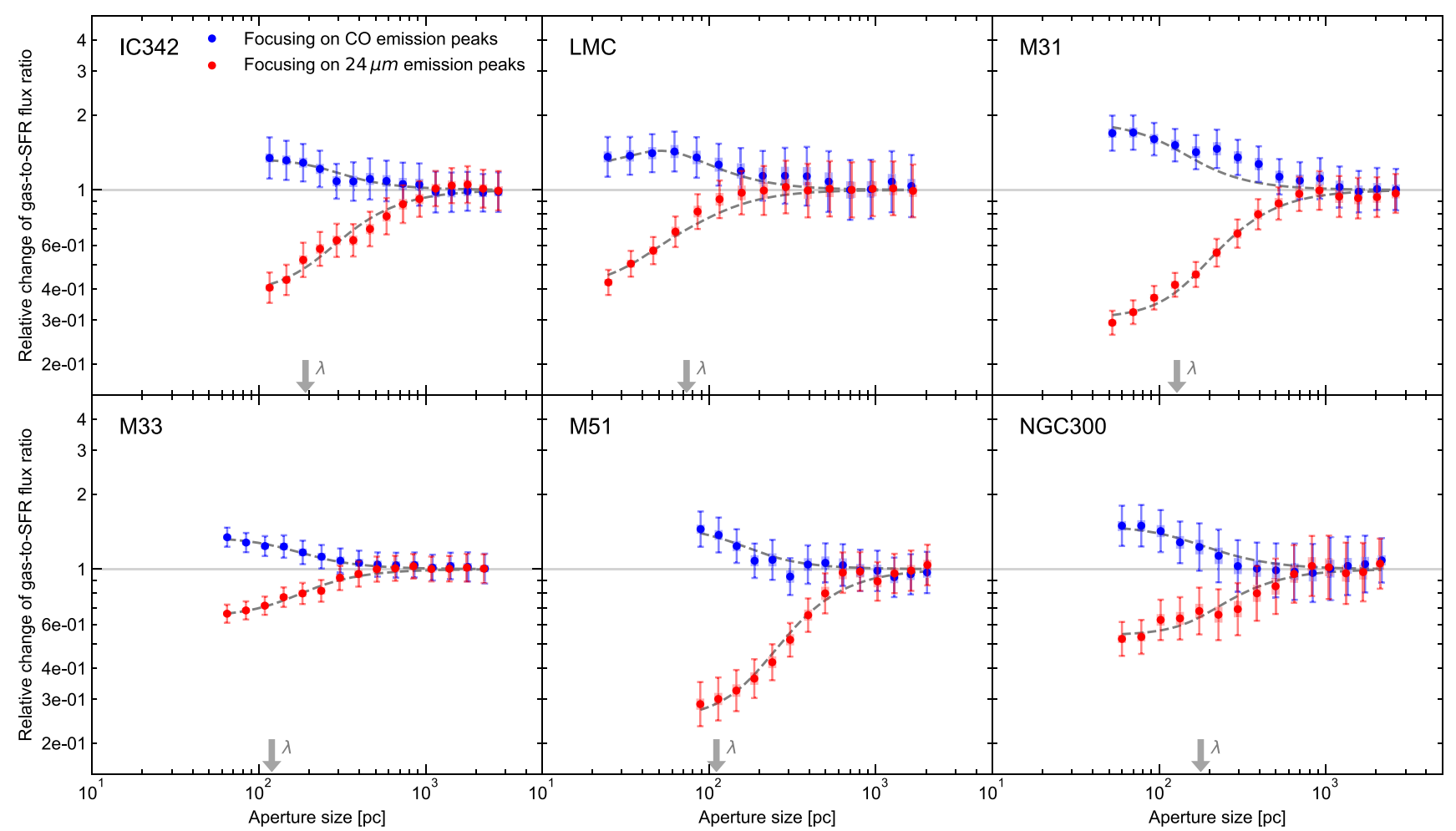

Figure 2. Relative change of the gas-to-SFR (CO-to-24 $\mu \mathrm{m}$ ) flux ratio compared to the galactic average as a function of the size of apertures placed on CO (blue) and $24 \mu \mathrm{m}$ (red) emission peaks. The error bars indicate $1 \sigma$ uncertainty on each individual data point whereas the shaded area is an effective $1 \sigma$ uncertainty taking into account the covariance between data points. The galactic average is shown as the solid horizontal line and the dashed line indicates our best-fitting model. The constrained region separation length $(\lambda)$ is indicated in each panel with the downward arrow and other constrained best-fitting parameters $\left(t_{\mathrm{fb}}, 24 \mu \mathrm{m}\right.$, and $\left.t_{24} \mu \mathrm{m}\right)$ are listed in Table 3.

where the luminosities have units of $\operatorname{erg~s}^{-1}$ and $L(24 \mu \mathrm{m})$ is expressed as $v L(v)$. We assume a typical uncertainty of 20 percent for the derived SFR. These conversion factors are only used to derive additional physical quantities such as the molecular gas surface density and the integrated star formation efficiency. We note that the exact values of these conversion factors, unless they vary spatially, do not affect our measurements of primary quantities, which are the durations of the successive phases of cloud evolution and star formation, nor do they affect the region separation length between independent regions.

\section{RESULTS}

\subsection{The molecular cloud lifecycle}

Here, we present our results from the application of our method to the maps of $\mathrm{CO}$ and $24 \mu \mathrm{m}$ emission presented in Section 2, as tracers of the molecular gas and the SFR for six nearby galaxies. Fig. 2 shows the gas-to-SFR flux ratios measured around gas and SFR tracer peaks, as a function of aperture size, together with our best-fitting model for each galaxy. Going towards smaller aperture sizes (from $\sim 1 \mathrm{kpc}$ to $\sim 50 \mathrm{pc}$ ), the measured flux ratios for both branches increasingly deviate from the galactic average, illustrating the spatial de-correlation between the gas and SFR tracer emission peaks. Table 3 summarizes the constrained best-fitting values from applying our analysis to the $\mathrm{H} \alpha$ and CO maps, as well as to the $24 \mu \mathrm{m}$ and $\mathrm{CO}$ maps. The first experiment allows us to measure $t_{\mathrm{CO}}$, which is then used as the reference time-scale for the second experiment (see Section 3). Table 3 also lists other physical quantities which can be derived from our measurements, such as the feedback outflow velocity $\left(v_{\mathrm{fb}}\right.$; see Section 4.1 .5$)$ and the integrated cloud-scale star formation efficiency $\left(\epsilon_{\mathrm{sf}}\right.$; see Section 4.1.4). In Fig. 3, we show an illustration of the evolutionary timelines of molecular clouds and star-forming regions in our galaxy sample. GMCs initially emit only in $\mathrm{CO}$, then in $24 \mu \mathrm{m}$ after the onset of star formation and finally in $\mathrm{H} \alpha$ when the star-forming regions become (partially) exposed.

\subsubsection{Feedback time-scale}

The use of $24 \mu \mathrm{m}$ emission enables us to take the heavily obscured phase of star formation into account, which cannot be done with the analysis of only $\mathrm{CO}$ and $\mathrm{H} \alpha$ emission. The duration of the embedded phase of massive star formation (i.e. feedback time-scale; $t_{\mathrm{fb}, 24 \mu \mathrm{m}}$ ), which continues until disruption of molecular clouds, is measured to be 2-7 Myr in our sample of galaxies. Our measurements suggest that molecular clouds spend $17-47$ per cent of their lifetime with massive stars embedded. For almost all of the galaxies in our sample (except M51), the measured $t_{\mathrm{fb}, 24 \mu \mathrm{m}}$ is $1-4 \mathrm{Myr}$ longer than the one obtained using $\mathrm{H} \alpha$ emission ( $t_{\mathrm{fb}, \mathrm{H} \alpha}$; see Table 3 ). This is expected, as $24 \mu \mathrm{m}$ is already detected during the heavily obscured phase of star formation, making it visible for a longer duration than $\mathrm{H} \alpha$, which is only detectable when massive stars have formed and surrounding gas and dust have been partially cleared out. By contrast, in the particular case of M51, we find $t_{\mathrm{fb}, 24 \mu \mathrm{m}}$ to be shorter than (or comparable within $1 \sigma$ uncertainty to) the feedback time-scale obtained using $\mathrm{H} \alpha$. We suspect that such a potentially unphysical measurement could be due 


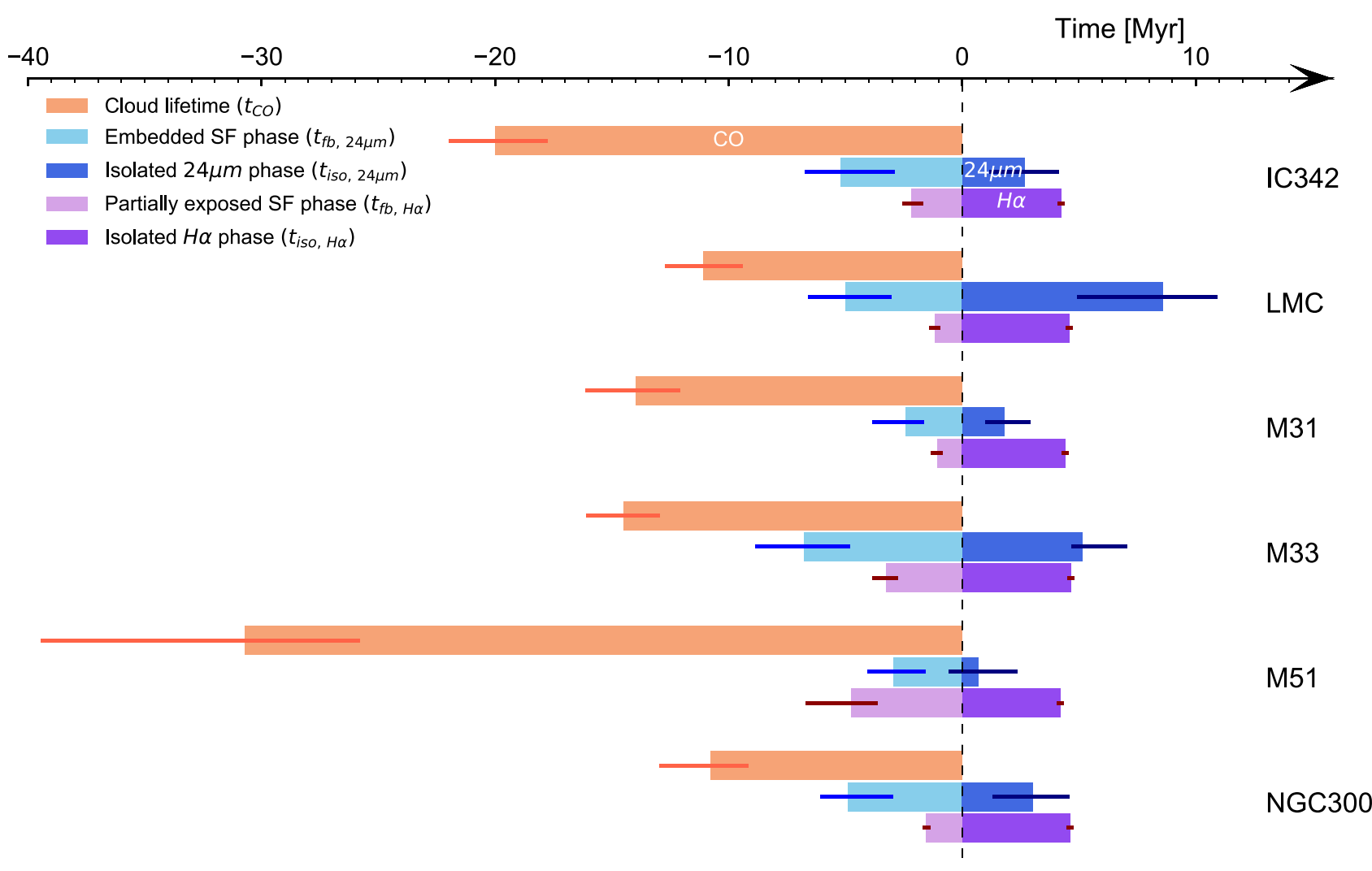

Figure 3. Timeline describing the evolution from molecular clouds to the embedded star-forming phase and then finally to exposed young stellar regions. The time during which $\mathrm{CO}$ is visible $\left(=t_{\mathrm{CO}}\right)$ is indicated in orange, the time during which $24 \mu \mathrm{m}$ and $\mathrm{H} \alpha$ are visible without $\mathrm{CO}$ are shown respectively in dark blue and dark purple. The time-scales for the feedback phase, during which both CO and SFR tracer emissions are observed co-spatially are shown in light blue (for $24 \mu \mathrm{m}$ ) and light purple (for $\mathrm{H} \alpha$ ). The corresponding $1 \sigma$ error bars are also indicated. We note that for M51 the feedback time-scale constrained using $24 \mu \mathrm{m}$ $\left(t_{\mathrm{fb}, 24 \mu \mathrm{m}}\right)$ could have been biased by deconvolution artefacts (see Section 4.1.1).

to artefacts in the $24 \mu \mathrm{m}$ map of M51 introduced by the deconvolution algorithm used to create the high resolution map (Dumas et al. 2011). These artefacts are clearly visible as dark rings around bright peaks in the spiral arms of the galaxy (see Fig. 1 and Appendix A), and may (or may not) make the inferred time-scales less accurate as discussed in Section 2.1.

The measured durations between the onset of embedded star formation and molecular cloud disruption (2-7 Myr), are comparable to the time it takes for the first supernova to explode. This is about $4 \mathrm{Myr}$ for a fully populated stellar initial mass function (Leitherer et al. 2014) and can be up to $20 \mathrm{Myr}$ when the initial mass function is stochastically sampled for the typical stellar region masses considered here (with almost no dependence on metallicity; Chevance et al. 2020a). The measured short feedback time-scales indicate that pre-supernova feedback such as photoionization and stellar winds are mostly responsible for the dispersal of molecular clouds. Our measurements of the feedback time-scale show a good agreement with the typical age of star clusters when they stop being associated with their natal GMCs both in the Milky Way and nearby galaxies (2-7 Myr; Lada \& Lada 2003; Whitmore et al. 2014; Hollyhead et al. 2015; Corbelli et al. 2017; Grasha et al. 2018, 2019). This is further discussed in Section 5.2. Radiation magnetohydrodynamic simulations of GMCs by Kim, Ostriker \& Filippova (2020) suggest a similar duration of the star formation and feedback time-scales (4-8 Myr). The measured duration for embedded star formation is somewhat shorter than the age spread of star clusters in the LMC (7-12 Myr) measured by Efremov \& Elmegreen (1998), on the scale of the mean radius of SFR tracer peaks $(\sim 10-50 \mathrm{pc})$. This is expected as the actual size of a starforming region is necessarily smaller than the size of a SFR tracer peak, which is limited by the spatial resolution of our maps. The agreement gets better if we only consider the age spreads measured among young stellar clusters (1-4 Myr; from star clusters with ages of $1-10 \mathrm{Myr})$.

\subsubsection{Duration of the $24 \mu \mathrm{m}$ emitting phase}

Across all galaxies in our sample, we find that the $24 \mu \mathrm{m}$ emission phase lasts for 4-14 Myr. For M33, we note that our measurement of $t_{24 \mu \mathrm{m}}$ is in very good agreement with the one from Corbelli et al. (2017), where $24 \mu \mathrm{m}$ emission is found to last for $\sim 10 \mathrm{Myr}$ after the onset of massive star formation by applying a cloud classification method to M33. The ratio between duration of visibility for $24 \mu \mathrm{m}$ and $\mathrm{H} \alpha$ emissions $\left(t_{24 \mu \mathrm{m}} / t_{\mathrm{H} \alpha}\right)$ ranges from 0.4 to 2.3 .

The $24 \mu \mathrm{m}$ emission does not originate only from embedded young massive stars, but also from late-type B stars and the interstellar radiation field, which make a non-negligible contribution to the dust heating (Draine \& Li 2007; Verley et al. 2009). The contribution of the sources not related to recent local massive star formation to the $24 \mu \mathrm{m}$ emission is more homogeneously spread in the galaxy compared to the more clustered young stellar population (e.g. Dale et al. 2007; Leroy et al. 2012) and results in an additional diffuse 
component of the $24 \mu \mathrm{m}$ emission on large scales. We separate this diffuse emission from the compact emission of young stellar regions by applying the filtering process described in Section 3. The fact that in most galaxies the end of the $24 \mu \mathrm{m}$ emission phase is before or similar to the end of the $\mathrm{H} \alpha$ emission phase (see Fig. 3) shows that this procedure effectively removed contamination from stellar populations not related to recent massive star formation. In Section 5.3, we discuss in more detail the effects of starless dust clumps illuminated by external radiation, and late-type B stars still preferentially located near their birth sites, which might not be removed by our filtering process.

We note that, while the duration of the isolated $\mathrm{H} \alpha$ emitting phase $\left(t_{\text {iso, } \mathrm{H} \alpha}=t_{\mathrm{H} \alpha}-t_{\mathrm{fb}, \mathrm{H} \alpha}\right.$ ) is almost constant in all the galaxies in our sample by construction (Haydon et al. 2020b), the duration of isolated $24 \mu \mathrm{m}$ emission $\left(t_{\text {iso }, 24 \mu \mathrm{m}}=t_{24 \mu \mathrm{m}}-t_{\mathrm{fb}, 24 \mu \mathrm{m}}\right)$ appears to vary across the sample ranging from $\sim 2 \mathrm{Myr}$ (excluding M51) to the end of the $\mathrm{H} \alpha$ emitting phase $\left(8.6_{-3.7}^{+2.3} \mathrm{Myr}\right)$. This isolated phase originates from stochastic heating of small dust grains in the $\mathrm{CO}$ dark clouds. We find this phase to be shorter for star-forming regions in more metalrich galaxies (see Figs 3 and 4). This is discussed in more detail in Section 4.3.

\subsubsection{Region separation length}

As visible in Fig. 2, gas and SFR tracer peaks are spatially decorrelated on small spatial scales, revealing that galaxies are made of regions that are independently undergoing evolution from molecular gas to stars. The spatial scale at which the gas-to-SFR ratio diverges from the galactic average (Fig. 2) is linked to the typical distance $\lambda$ between independent regions. We find that $\lambda$ ranges from 70 to $190 \mathrm{pc}$ for the galaxies in our sample when considering the $24 \mu \mathrm{m}$ and $\mathrm{CO}$ emission maps. For M51, we do not sufficiently resolve the region separation length and are only able to obtain an upper limit of $\lambda$ (see Section 5.1.1). For the other galaxies, we find that $\lambda$ derived using $24 \mu \mathrm{m}$ maps and $\mathrm{H} \alpha$ maps agree to within the formal uncertainties.

While the physical mechanisms that set the region separation length remain debated, a similarity between the region separation length and the gas disc scale height has been reported by Kruijssen et al. (2019) in NGC 300, suggesting that the depressurization of $\mathrm{H}$ II regions along the direction perpendicular to the galactic disc might be responsible for this characteristic length. Furthermore, the measured values of $\lambda$ are comparable to the thickness of the vertical distribution of star-forming regions undergoing the earliest stages of evolution (150-200 pc) observed using Spitzer IRAC $8 \mu \mathrm{m}$ maps of edge-on spiral galaxies (NGC 891 and IC 5052; Elmegreen \& Elmegreen 2020), to the thickness of the molecular disc measured with CO emission (Scoville et al. 1993; Yim et al. 2014; Heyer \& Dame 2015; Patra 2020; Yim et al. 2020), and also to the amplitude of the oscillation seen in the Radcliffe Wave recently discovered in the vicinity of the Sun (Alves et al. 2020). Finally, the region separation length roughly coincides with the spatial wavelength of velocity corrugations in NGC 4321 (Henshaw et al. 2020), indicating that it matches the scale on which the molecular interstellar medium is reorganized by cloud-scale matter flows. Further investigation is needed to verify quantitatively whether the correlation observed by Kruijssen et al. (2019) holds more generally in nearby galaxies.

\subsubsection{Star-formation efficiency}

The SFR surface density $\left(\Sigma_{\mathrm{SFR}}\right)$ corresponds to the mass of newly formed stars inferred for a given SFR tracer, divided by that SFR tracer's emission time-scale. Similarly, the rate of molecular gas formation can be expressed as $\Sigma_{\text {gas }} / t_{\mathrm{CO}}$, where $\Sigma_{\text {gas }}$ is the surface density of molecular gas and the $t_{\mathrm{CO}}$ is the time-scale over which molecular gas assembles and form stars. By dividing these two rates, the time-averaged star formation efficiency per star-forming event $\left(\epsilon_{\text {sf }}\right)$ can be computed as:

$\epsilon_{\mathrm{sf}}=\frac{t_{\mathrm{CO}} \Sigma_{\mathrm{SFR}}}{\Sigma_{\mathrm{gas}}}$.

When calculating $\Sigma_{\text {gas }}$, we only consider the compact CO emission, after the filtering of diffuse emission (see Section 3), which is also consistent with the flux we use to determine $t_{\mathrm{CO}}$. By doing this, we selectively include the $\mathrm{CO}$ emission that participates in the massive star formation process while excluding emission that is likely to originate from diffuse molecular gas and small clouds. The filtering process removes 10 per cent to 50 per cent of the $\mathrm{CO}$ emission from the unfiltered maps. However, $\Sigma_{\mathrm{SFR}}$ is calculated using the total SFR, obtained by combining $\mathrm{H} \alpha$ and $24 \mu \mathrm{m}$ emission to account for the effect of internal extinction (see Section 2). We note that our assumption implies that we attribute all of the diffuse emission in SFR tracer maps to recent massive star formation (e.g. leakage of ionizing photons from HII regions). This ignores the fact that diffuse emission may also originate from mechanisms that are not related to recent massive star formation, such as diffuse ionized gas created by shocks and evolved post-asymptotic giant branch stars, as well as infrared emission powered by older stellar populations, which are known to have a relatively minor contribution to the dust heating (Nersesian et al. 2020). Under these conditions, we measure a low star formation efficiency per star-forming event in our sample of galaxies with $\epsilon_{\mathrm{sf}}=0.7-6.8$ per cent. This is consistent with previous measurements in these galaxies using $\mathrm{H} \alpha$ as a tracer of recent star formation and other wavelengths such as GALEX FUV and WISE $22 \mu \mathrm{m}$ (Leroy et al. 2012, 2019) to estimate the global SFR (Chevance et al. 2020c; Hygate 2020; Ward et al. 2020a). We note that for NGC 300, we find $\epsilon_{\text {sf }}$ to be slightly higher (but compatible within $1 \sigma$ uncertainty) than that measured in Kruijssen et al. (2019). The difference is because Kruijssen et al. (2019) only considered $\mathrm{H} \alpha$ emission when calculating the global SFR.

We also compare our measurements for $\epsilon_{\mathrm{sf}}$ to the fraction of gas converted into stars per gravitational free-fall time, which is expressed as $\epsilon_{\mathrm{ff}}=t_{\mathrm{ff}} \Sigma_{\mathrm{SFR}} / \Sigma_{\text {gas }}$ and measured by Leroy et al. 2017, Utomo et al. 2018 and Schruba, Kruijssen \& Leroy (2019) for most of the galaxies in our sample. We find that our measurements for the LMC, M31, M33, and NGC 300 are somewhat ( $\leq 4.0$ per cent) higher than the star formation efficiency per free-fall time $\left(\epsilon_{\mathrm{ff}}\right)$ measured by Schruba et al. (2019), which are 2.5 per cent, 0.7 per cent, 1.5 per cent, and 1.2 per cent, respectively. Because the cloud lifetime in these galaxies is similar to the free-fall time-scale (Schruba et al. 2019), this difference is mostly due to the fact that we measure gas surface density from the diffuse emission filtered CO map, in order to calculate the fraction of compact clouds turning into stars. For M51, the difference becomes more significant $\left(\epsilon_{\mathrm{ff}}=0.3-0.36\right.$ per cent; Leroy et al. 2017; Utomo et al. 2018) and is because cloud lifetime is almost five times the free-fall time-scale.

\subsubsection{Feedback velocity}

As a result of the energetic feedback from young massive stars, $\mathrm{CO}$ emission becomes rapidly undetectable after the onset of star formation. This is most likely due to a phase and density change of the neighbouring medium through kinetic dispersal, ionization, and photodissociation. We combine the time-scale over which molecular 

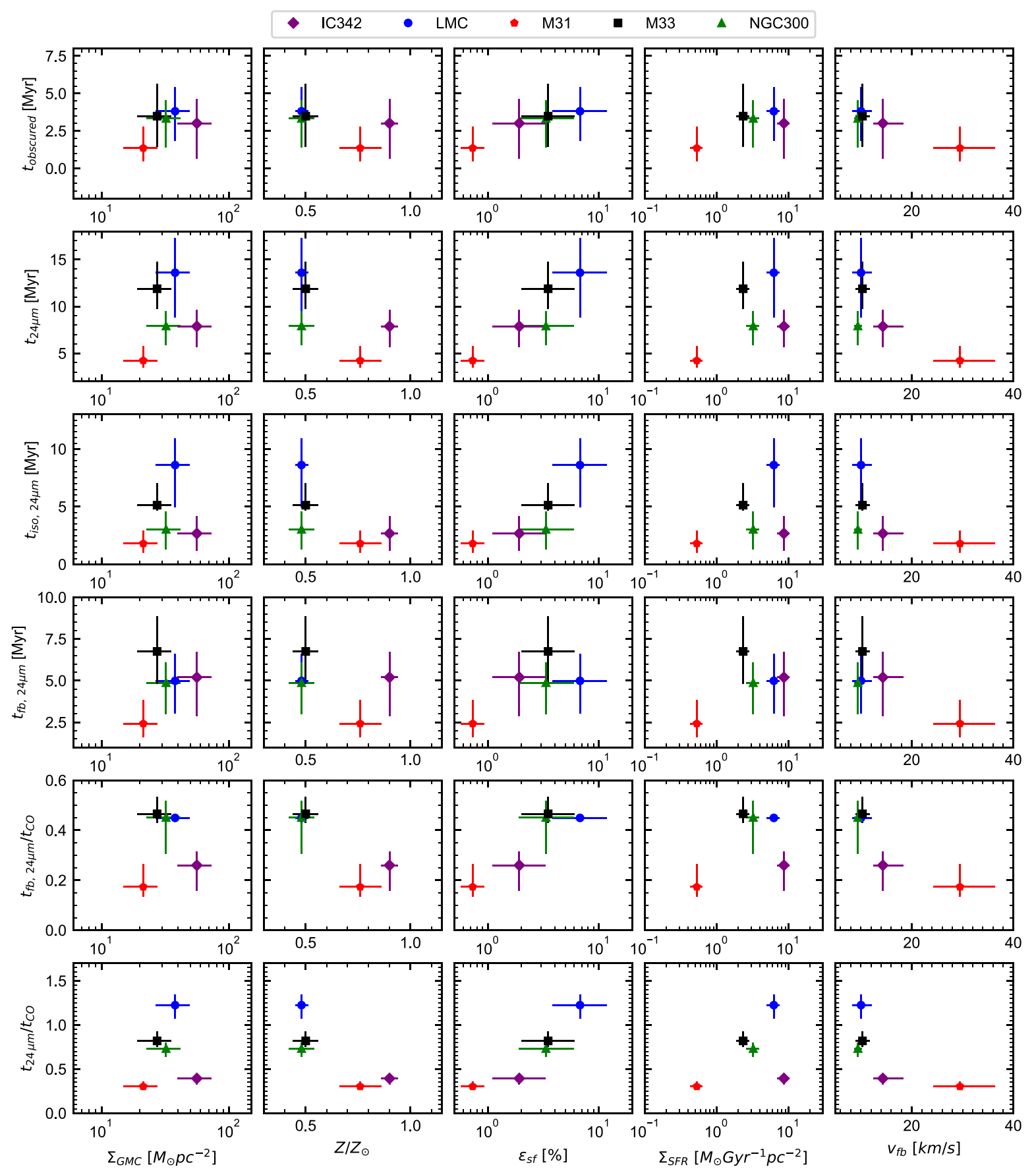

Figure 4. In the top four rows, our measurements of the duration of the heavily obscured phase $\left(t_{\mathrm{obscured}}=t_{\mathrm{fb}, 24 \mu \mathrm{m}}-t_{\mathrm{fb}, \mathrm{H} \alpha}\right)$, the $24 \mu \mathrm{m}$ emitting phase

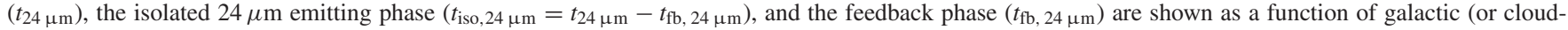
scale) properties, i.e. the mass-weighted mean molecular gas surface density of GMCs $\left(\Sigma_{\text {GMC }}\right)$ measured in Schruba et al. (2019) and Schruba et al. (2021a), metallicity $\left(Z / Z_{\odot}\right)$, star formation efficiency $\left(\epsilon_{\mathrm{sf}}\right)$, SFR surface density $\left(\Sigma_{\mathrm{SFR}}\right)$, and the feedback velocity $\left(v_{\mathrm{fb}}\right)$. The bottom two rows show the ratios of the feedback time-scale and the $24 \mu \mathrm{m}$ emitting phase to the cloud lifetime $\left(t_{\mathrm{fb}}, 24 \mu \mathrm{m} / t_{\mathrm{CO}}\right.$, and $\left.t_{24 \mu \mathrm{m}} / t_{\mathrm{CO}}\right)$, as a function of the same galactic properties.

clouds are disrupted by feedback, $t_{\mathrm{fb}}$, with the characteristic size of the clouds detected in $\mathrm{CO}, r_{\mathrm{CO}}$, to define the feedback velocity as $v_{\mathrm{fb}}=r_{\mathrm{CO}} / t_{\mathrm{fb}}$. The size of the cloud is measured in our method by fitting a Gaussian profile to the surface density contrast between the peak and the background, and ranges between 10 and $40 \mathrm{pc}$ for the sample of our galaxies. The velocity represents the speed of the kinetic removal of molecular gas or the phase transition front, depending on the nature of the dispersal mechanism. We use here $t_{\mathrm{fb}, \mathrm{H} \alpha}$ to define $v_{\mathrm{fb}}$, assuming that disruption of the molecular gas starts with the young stellar population becoming partially exposed, whereas the $24 \mu \mathrm{m}$ is emitted even during the heavily obscured phase where the expansion of the HII region has not yet begun and during which gas accretion on to the cloud potentially continues. 
The derived $v_{\mathrm{fb}}$ ranges between 9 and $30 \mathrm{~km} \mathrm{~s}^{-1}$, which is comparable to the measurements of expansion velocities of nearby $\mathrm{H}$ II regions. For example, the expansion velocities of five $\mathrm{H}$ II regions in NGC 300 are measured to be in the range of 5-30 $\mathrm{km} \mathrm{s}^{-1}$ (McLeod et al. 2020). Similar values are also found for $\mathrm{H}$ II regions in the LMC (Nazé et al. 2001, 2002; McLeod et al. 2019) and the Milky Way (Murray \& Rahman 2010; Barnes et al. 2020). Numerical simulations of star-forming regions (e.g. Kim, Kim \& Ostriker 2018) also support this range of values.

\subsection{Heavily obscured phase of star formation}

In order to probe the earliest phase of star formation, which is only associated with $24 \mu \mathrm{m}$ emission and not with $\mathrm{H} \alpha$ because of strong attenuation provided by the surrounding gas, we measure the duration of the heavily obscured phase of star formation as the difference between the feedback time-scale for $24 \mu \mathrm{m}$ emission (i.e. the total duration of the embedded phase of star formation) and the one for $\mathrm{H} \alpha$ emission (i.e. the duration of the partially exposed phase of star formation): $t_{\mathrm{obscured}}=t_{\mathrm{fb}, 24 \mu \mathrm{m}}-t_{\mathrm{fb}, \mathrm{H} \alpha}$. We omit M51 here because the insufficient resolution of the $24 \mu \mathrm{m}$ map only allows for the determination of an upper limit on $t_{\mathrm{fb}, 24 \mu \mathrm{m}}$ (see Section 5.1.1) and the presence of deconvolution artefacts (see Section 2.1) could have biased our measurements, especially for time-scale-related quantities. However, we retain M51 in our sample at large, to show our (unsuccessful) attempt in applying the method to a $24 \mu \mathrm{m}$ emission map that has been created using the HiRes deconvolution algorithm (Backus et al. 2005). Across the rest of our galaxy sample, we find $t_{\text {obscured }}=3.0 \pm 0.9 \mathrm{Myr}$, with a full range of $1.4-3.8 \mathrm{Myr}$ (see Fig. 4). The measured duration is comparable to age estimates of heavily obscured star clusters in the Milky Way (0.5-3 Myr; see Lada \& Lada 2003, and references therein). A similar duration of the highly embedded star-forming phase has recently been reported by Elmegreen \& Elmegreen (2019), Elmegreen \& Elmegreen (2020), where such a phase is suggested to last for $1-2 \mathrm{Myr}$ based on the mass measurement of star-forming cores in nearby spiral galaxies using $8 \mu \mathrm{m}$ emission. The measurement of $2.4 \mathrm{Myr}$ for the heavily obscured phase using a cloud classification method by Corbelli et al. (2017) also supports the values we obtain here (see more in Section 5.2).

\subsection{Relation with environmental properties}

We now explore potential environmental dependences of the durations of the successive phases of cloud evolution and star formation. In Fig. 4, our measurements of the durations of the heavily obscured phase $\left(t_{\text {obscured }}\right)$, the total $24 \mu \mathrm{m}$ emission phase $\left(t_{24 \mu \mathrm{m}}\right)$, the isolated $24 \mu \mathrm{m}$ emission phase $\left(t_{\text {iso, } 24 \mu \mathrm{m}}=t_{24 \mu \mathrm{m}}-t_{\mathrm{fb}, 24 \mu \mathrm{m}}\right)$, and the feedback phase $\left(t_{\mathrm{fb}, 24 \mu \mathrm{m}}\right)$ are shown in the top four rows as a function of local cloud-scale and galactic properties such as the mass-weighted mean molecular gas surface density of GMCs $\left(\Sigma_{\mathrm{GMC}}\right.$; measured by Schruba et al. 2021a for IC342 and Schruba et al. 2019 for the other galaxies), metallicity (relative to solar metallicity), integrated star formation efficiency per star formation event $\left(\epsilon_{\mathrm{sf}}\right)$, SFR surface density $\left(\Sigma_{\mathrm{SFR}}\right)$, and the feedback velocity $\left(v_{\mathrm{fb}}\right){ }^{4}$

In order to look for correlations with these environmental properties, we use LINMIX (Kelly 2007), a Bayesian method accounting

\footnotetext{
${ }^{4}$ The measurement of $\Sigma_{\mathrm{GMC}}$ for IC 342 is calculated excluding the five most massive clouds (with mass $>2 \times 10^{7} \mathrm{M}_{\odot}$ ), which contribute 13 percent of the total mass contained in molecular clouds. Including these clouds would not change our conclusion.
}

for measurement errors in linear regression. We do not find any statistically significant trend, which is defined to exist when the correlation coefficient is positive or negative with 95 per cent probability. While this is not shown in Fig. 4, we also found no statistically significant correlation with the galaxy-averaged molecular gas surface density $\left(\Sigma_{\text {gas }}\right)$. However, we note that $t_{24 \mu \mathrm{m}}$ and $t_{\text {iso, } 24 \mu \mathrm{m}}$ appear to be somewhat shorter towards increasing metallicity and decreasing star formation efficiency. While it is difficult to distinguish what is driving this trend, as metallicity and star formation efficiency are correlated in our sample of galaxies, we suspect that metallicity could be the primary driver of this trend. Indeed, winds from massive stars become more energetic with increasing metallicity (Maeder 1992), resulting in a faster dispersal of the surrounding gas and decay of $t_{24 \mu \mathrm{m}}$ emission. We note that one might expect to see longer (isolated) $24 \mu \mathrm{m}$ emitting time-scale for metal-rich galaxies since the dust-togas ratio correlates with metallicity (Rémy-Ruyer et al. 2014). This is not what we find here, which can be explained by our use of relative changes of gas-to-SFR tracer flux ratio compared to the galactic average, instead of using absolute flux ratios, when constraining the timeline of GMC evolution.

The relative fractions of $t_{\mathrm{fb}, 24 \mu \mathrm{m}}$ and $t_{24 \mu \mathrm{m}}$ compared to $t_{\mathrm{CO}}$ are plotted against galactic properties in the bottom two rows of Fig. 4. We again see an anticorrelation of these durations with metallicity and a correlation with star formation efficiency, while no statistically significant trend is found with respect to galactic properties using the same regression analysis (Kelly 2007). Our results (bottom row) show that clouds in the LMC, M33, and NGC 300 spend a larger fraction of their lifetime with embedded massive star formation ( $\sim 40$ per cent) compared to clouds in IC 342 and M31 ( 20 per cent), with an average of 36 per cent across our sample of galaxies.

\section{DISCUSSION}

\subsection{Robustness of the results}

\subsubsection{Satisfaction of guidelines in Kruijssen et al. (2018)}

We verify here that our analysis satisfies the requirements listed in section 4.4 of Kruijssen et al. (2018). Satisfaction of these criteria indicates that the constrained parameters $t_{24 \mu \mathrm{m}}, t_{\mathrm{fb}, 24 \mu \mathrm{m}}$, and $\lambda$ are measured with an accuracy of at least 30 per cent. For the analysis using $\mathrm{H} \alpha$ as an SFR tracer, we only check the accuracy for IC 342 and M31, because the measurements for other galaxies have already been validated by previous studies (see Kruijssen et al. 2019; Chevance et al. 2020c; Hygate 2020; Ward et al. 2020a).

(i) The duration of gas and stellar phases should always differ less than one order of magnitude. This condition is satisfied by $\left|\log _{10}\left(t_{\mathrm{H} \alpha} / t_{\mathrm{CO}}\right)\right| \leq 0.5$ for IC 342 and M31, and $\left|\log _{10}\left(t_{24 \mu \mathrm{m}} / t_{\mathrm{CO}}\right)\right| \leq$ 0.92 for all the galaxies in our sample, where the difference between $t_{24 \mu \mathrm{m}}$ and $t_{\mathrm{CO}}$ is the largest in M51 while those for other galaxies are $\left|\log _{10}\left(t_{24 \mu \mathrm{m}} / t_{\mathrm{CO}}\right)\right| \leq 0.52$.

(ii) For almost all of the galaxies (except M51), we measure $\lambda \geq 1.6 l_{\text {ap, } \min }$, which ensures that the region separation length is sufficiently resolved by our observations. For M51, we measure $\lambda=$ $1.2 l_{\mathrm{ap}, \mathrm{min}}$, implying that $t_{24 \mu \mathrm{m}}$ can be constrained with sufficient accuracy, but only upper limits can be derived for $\lambda$ and $t_{\mathrm{fb}, 24 \mu \mathrm{m}}$.

(iii) The number of identified emission peaks is always above 35 , both in the $\mathrm{CO}$ and the $24 \mu \mathrm{m}$ emission maps, as well as in the $\mathrm{H} \alpha$ maps of IC 342 and M31.

(iv) The measured gas-to-SFR flux ratios focussing on gas (SFR tracer) peaks should never be below (above) the galactic average. 
This condition may not always be true in the presence of a diffuse emission reservoir. As visible in Fig. 2, this criterion is satisfied after we filter out the large-scale diffuse emission in both tracer maps.

(v) In order to perform accurate measurements, we require the global star formation history to not vary more than 0.2 dex during the duration of the whole evolutionary cycle (ranging from $\sim 15$ to 35 Myr for our sample) when averaged over time intervals of width $t_{24 \mu \mathrm{m}}$ or $t_{\mathrm{CO}}$. Using multiwavelength data of the LMC and synthetic stellar population models, Harris \& Zaritsky (2009) studied the star formation history in the LMC and found a roughly consistent SFR during the duration of the whole evolutionary cycle. The criterion is also satisfied in M33 and NGC 300 as shown by Kang et al. (2012), Kang et al. (2016) using chemical evolution models to reconstruct the star formation rate history. Using data from the PHAT survey, the recent star formation history of M31 is confirmed to be quiescent without significant variations (Lewis et al. 2015; Williams et al. 2017). By performing spectral energy distribution (SED) fitting to the multiwavelength data of M51, the SFR in M51 is also measured to be roughly constant for the last $100 \mathrm{Myr}$ (Eufrasio et al. 2017). The star formation history of IC 342 is not known. However, we do not expect it to experience significant variations in the global star formation rate during the last $\sim 30 \mathrm{Myr}$ when averaged over $t_{24 \mu \mathrm{m}}=8 \mathrm{Myr}$. In addition, we mask the starburst nucleus of this galaxy, which experienced a major burst of star formation $\sim 60 \mathrm{Myr}$ ago (Böker, van der Marel \& Vacca 1999).

(vi) Each independent region should be detectable at given sensitivity in both tracers at some point in their life. In order to check if this condition is satisfied in our sample of galaxies, we first calculate the minimum star-forming region mass expected to form from the detected molecular clouds by multiplying the star formation efficiency obtained in our method (see Table 3 ) by the $5 \sigma$ pointsource sensitivity limit of the CO map. We then compare this mass to the mass of the stellar population required to provide an ionizing radiation luminosity that matches the $5 \sigma$ sensitivities of $24 \mu \mathrm{m}$ and $\mathrm{H} \alpha$ maps on the scale of individual star-forming regions $(\lambda)$. We use the Starburst99 model (Leitherer et al. 1999) to calculate the initial mass of the stellar population assuming stars formed instantaneously 5 Myr ago (similarly to the $\mathrm{H} \alpha$ emitting time-scale). Since Starburst 99 only provides models for the $\mathrm{H} \alpha$ luminosity as a function of the age of the stellar population at different metallicities, we use the relation from Kennicutt \& Evans (2012, and references therein), $\log \mathrm{SFR}=\log \left(L_{\mathrm{H} \alpha}\right)-41.27=\log \left(\nu L_{24 \mu \mathrm{m}}\right)-42.69$, to obtain a similar estimation of the $24 \mu \mathrm{m}$ luminosity. We find that the minimum mass of the stellar population obtained from CO maps agrees well with that obtained from $24 \mu \mathrm{m}$ and $\mathrm{H} \alpha$ maps (ranging from 100 to $5000 \mathrm{M}_{\odot}$ for the galaxies in our sample), suggesting that the sensitivity of the gas and SFR tracer maps are well-matched and the faintest $\mathrm{CO}$ peak is likely to evolve into the faintest $\mathrm{H}$ II region. In principle, clouds can disperse dynamically before forming massive stars and then reassemble. In this case, the time spent before GMC dispersal would be added by our method to the measured lifetimes of clouds that do form massive stars. However, Kruijssen et al. (2019) and Chevance et al. (2020c) show that this is unlikely to happen because the clouds are found to live only for about one dynamical time-scale, not leaving enough time for clouds to disperse and recollapse before forming massive stars.

Most of our measurements of $t_{24 \mu \mathrm{m}}$ and $\lambda$ for all the galaxies in our sample as well as the $t_{\mathrm{CO}}$ and $\lambda$ for IC 342 and M31 with $\mathrm{H} \alpha$ as an SFR tracer are validated by satisfying the conditions listed above. The only exception is for M51, where we neither have sufficient resolution to accurately constrain the region separation length nor the feedback time-scale. Only upper limits can be obtained for these values. In order to determine whether our measurements are reliable for $t_{\mathrm{fb}, 24 \mu \mathrm{m}}$ for all the galaxies in our sample and $t_{\mathrm{fb}, \mathrm{H} \alpha}$ for IC 342 and M31, we use four additional criteria listed in Kruijssen et al. (2018). To do so, we first introduce the filling factor of SFR tracer or gas peaks as $\zeta=2 r / \lambda$, where $r$ is the mean radius of the corresponding peaks. This parameter characterizes how densely the peaks are distributed in a map.

i) If peaks are densely distributed and potentially overlapping with each other, the density contrast used for peak identification $\left(\delta \log _{10} \mathcal{F}\right)$ should be small enough to identify adjacent peaks. We compare in Fig. 5 our values for $\delta \log _{10} \mathcal{F}$ with the upper limit prescribed by Kruijssen et al. (2018) and show that our choice enables the appropriate detection of neighbouring peaks, even in densely populated environments.

ii) For an accurate measurement of the feedback time-scale, contamination by neighbouring peaks should be small enough. Indeed, spatial overlap of neighbouring peaks due to low resolution or blending can be falsely attributed to a temporal overlap, therefore artificially increasing the duration of the measured feedback timescale. In this case, only an upper limit on the feedback time-scale can be determined. In Fig. 5, we compare the analytical prescription of Kruijssen et al. (2018) with our measurements of $t_{\mathrm{fb}} / \tau$ and average $\zeta$, where $\tau$ is the total duration of the whole evolutionary cycle $\left(\tau=t_{\mathrm{CO}}+t_{24 \mu \mathrm{m}}-t_{\mathrm{fb}, 24 \mu \mathrm{m}}\right)$. The average $\zeta$ is obtained by weighting the filling factors for gas and SFR tracer peaks by their corresponding time-scales. We find that this condition is not satisfied for $t_{\mathrm{fb}, 24 \mu \mathrm{m}}$ in M51. Only upper limits on this quantity can be determined (see Table 3 ).

iii) Fig. 5 shows that the conditions $t_{\mathrm{fb}}>0.05 \tau$ and $t_{\mathrm{fb}}<0.95 \tau$ are verified for all galaxies.

iv) Similarly to condition (v), the SFR should not vary more than 0.2 dex during the entire timeline when averaged over the width of feedback time-scale. This condition is also satisfied using the same reasoning, as stated in (v) above.

v) After masking obvious blended regions such as galactic centres, visual inspection of the maps does not reveal abundant blending (Fig. 1).

Overall, we find that our measurements are reliable except for $\lambda$ and $t_{\mathrm{fb}, 24 \mu \mathrm{m}}$ in M51. These two measurements should formally be considered as upper limits as they do not satisfy conditions (ii) and (viii). However, we note that the deconvolution artefacts present in the $24 \mu \mathrm{m}$ map of M51 may (or may not) bias the feedback timescale and therefore the value we obtain as the upper limit should be considered uncertain.

\subsubsection{Effect of spatial resolution and inclination on the measured quantities}

In order to test the potential effect of spatial resolution on our measurements, we have degraded the resolution of the $\mathrm{CO}$ and $24 \mu \mathrm{m}$ emission maps of NGC 300 to the coarsest resolution among our galaxy sample (107 pc, see Table 1) and repeated the analysis described in Section 3. We choose NGC 300 for this test as it does not require bright star-forming regions to be masked like in the LMC and M33, making the application of the method more straightforward. We find that time-scales $\left(t_{24 \mu \mathrm{m}}=8.4_{-2.1}^{+3.8} \mathrm{Myr} ; t_{\mathrm{fb}, 24 \mu \mathrm{m}}=4.8_{-2.6}^{+1.5} \mathrm{Myr}\right)$ and the mean separation length $\left(\lambda=217_{-112}^{+145} \mathrm{pc}\right)$ measured at a different spatial resolution are consistent within $1 \sigma$ uncertainties with the results of Table 3, confirming previous resolution tests on 

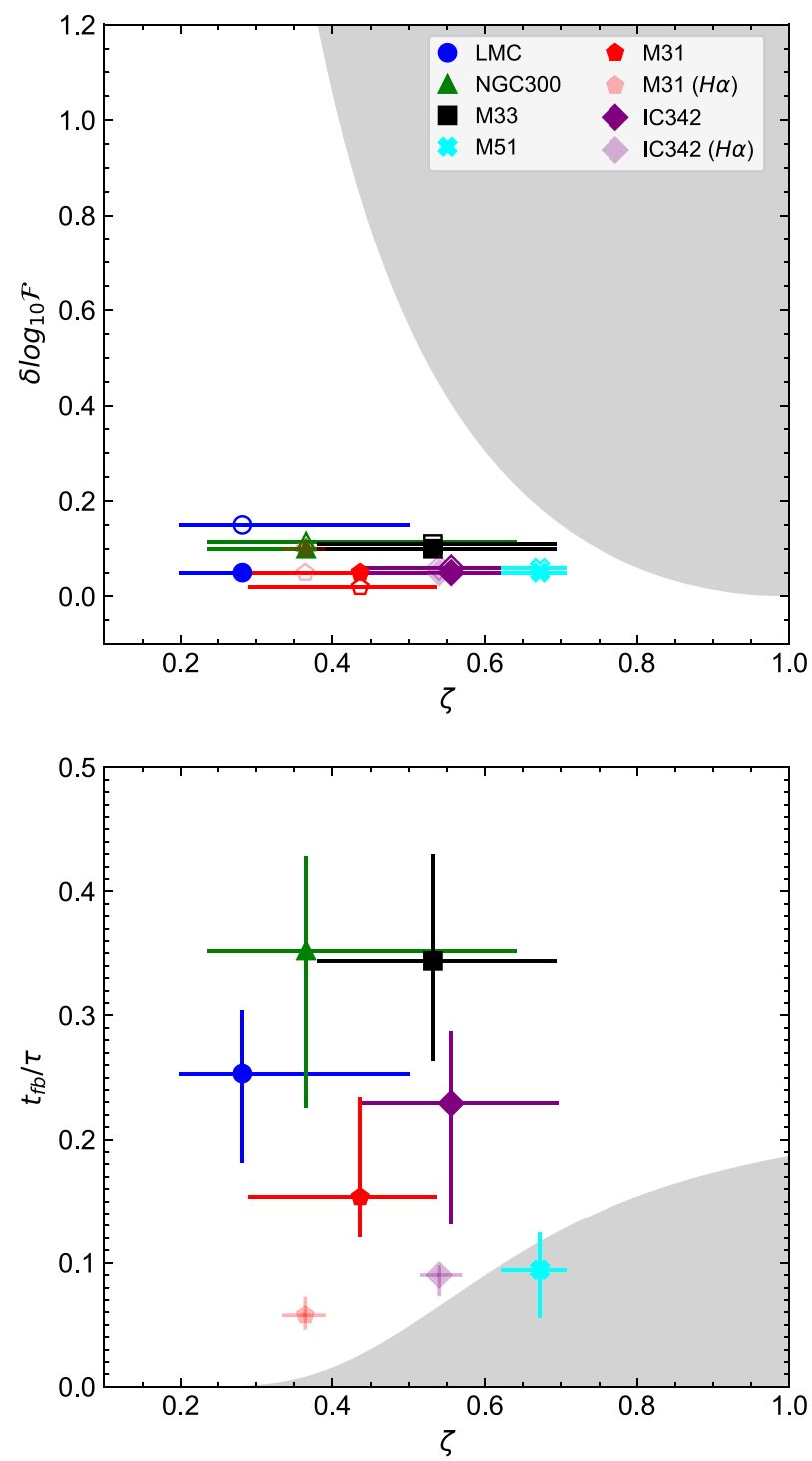

Figure 5. Effects of blending on the feedback time-scale measurements. The top panel shows the adopted density contrasts $\left(\delta \log _{10} \mathcal{F}\right)$ used for peak identification in each $24 \mu \mathrm{m}$ (filled symbols) and $\mathrm{CO}$ (open symbols) emission map, as a function of the average filling factor $\zeta$. The transparent markers for IC 342 and M31 indicate the adopted $\delta \log _{10} \mathcal{F}$ and measured $\zeta$ for the analysis with $\mathrm{H} \alpha$ as SFR tracer. The shaded area indicates the region of the parameter space where peak identification is affected by blending (Kruijssen et al. 2018). Our results are well outside of the shaded area, confirming that we adopt small enough $\delta \log _{10} \mathcal{F}$ to identify adjacent peaks even in maps with high-filling factor. The bottom panel shows the ratio between the feedback time-scale $\left(t_{\mathrm{fb}}\right)$ and the total duration of the whole evolutionary cycle $(\tau)$ as a function of the average filling factor. The grey shaded area indicates the region of the parameter space where the contamination by neighbouring peaks affects the measurement of the feedback time. As a result, only an upper limit can be determined for $t_{\mathrm{fb}, 24 \mu \mathrm{m}}$ in M51, whereas accurate measurements can be made for all other galaxies.

simulated galaxies (Kruijssen et al. 2018) and on NGC 300 (Kruijssen et al. 2019) using $\mathrm{H} \alpha$ as a SFR tracer.

We have also examined the possible effect of inclination on our measurements by repeating the analysis on $\mathrm{CO}$ and $24 \mu \mathrm{m}$ emission maps of NGC 300, which have been artificially inclined further to match the highest inclination angle among our galaxy sample
(M31; $\left.i=77.7^{\circ}\right)$. We find that measured time-scales $\left(t_{24 \mu m}=\right.$ $\left.10.4_{-2.0}^{+2.7} \mathrm{Myr} ; t_{\mathrm{fb}, 24 \mu \mathrm{m}}=3.9_{-0.9}^{+1.0} \mathrm{Myr}\right)$ and the mean separation length $\left(\lambda=192_{-60}^{+130} \mathrm{pc}\right)$ agree within $1 \sigma$ uncertainties with those of NGC 300 from Table 3. This is already expected from a similar test performed by Kruijssen et al. (2018) using simulated galaxies, where our method has been shown to provide reliable measurements even for a highly inclined galaxy as long as independent starforming regions are sufficiently resolved $\left(\lambda \geq 1.5 l_{\mathrm{ap}, \min }\right.$; see (ii) in Section 5.1.1).

\subsection{Comparison with other works}

The duration of the embedded star-forming phase has been measured in M33 by Corbelli et al. (2017), using IRAM CO data and the midinfrared source catalog created by Sharma et al. (2011). In their work, GMCs and star-forming regions are classified into different evolutionary stages based on the presence of CO emission and SFR tracers such as $24 \mu \mathrm{m}$ and $\mathrm{H} \alpha$ or $\mathrm{UV}$ emission. The clouds are defined to be in an inactive stage when no sign of star formation is detected, an embedded star-forming phase when $\mathrm{CO}$ emission is observed in association with $24 \mu \mathrm{m}$ but without associated $\mathrm{H} \alpha$ or FUV emission. The region is defined to be at an exposed star-forming phase when $\mathrm{H} \alpha$ or FUV emission becomes visible.

The age estimates of the exposed star-forming regions (referred to as C-type in Corbelli et al. 2017) from SED fitting are available in Sharma et al. (2011), and are obtained using photometric data at various wavelengths simultaneously, such as $\mathrm{UV}, \mathrm{H} \alpha$, and $24 \mu \mathrm{m}$. The age of the C-type phase corresponds to the time it takes for the cloud to evolve from the end of the heavily obscured phase of star formation (observed with $24 \mu \mathrm{m}$ but without $\mathrm{H} \alpha$ ) to the end of the exposed young stellar region phase (both $24 \mu \mathrm{m}$ and $\mathrm{H} \alpha$ are observed). This duration therefore corresponds to $t_{24 \mu \mathrm{m}}-t_{\mathrm{obscured}}$ in our analysis. Corbelli et al. (2017) find that the C-type phase in M33 lasts for $8 \mathrm{Myr}$ (without quoted uncertainty), which is in excellent agreement with our measurement of $8.4_{-3.0}^{+3.6}$ Myr. For the duration of the heavily obscured phase of star formation ( $\mathrm{CO}$ and $24 \mu \mathrm{m}$ emission without $\mathrm{H} \alpha$; referred to as B-type), Corbelli et al. (2017) find $2.4 \mathrm{Myr}$, which is similar to the duration we measure, not only for M33 (3.5 $5_{-1.9}^{+1.2} \mathrm{Myr}$ ), but for most of the galaxies in our sample (1.4-3.8 Myr; see Section 4.2). Lastly, as for the duration of the inactive phase (referred to as A-type), we measure $t_{\mathrm{CO}}-t_{\mathrm{fb}, 24 \mu \mathrm{m}}=$ $7.7_{-1.7}^{+1.4} \mathrm{Myr}$, which is somewhat longer than the measurement of 4 Myr from Corbelli et al. (2017). However, given the uncertainties in age estimates using SED fitting (on the order of $0.1 \mathrm{dex}$ ) and the absence of any uncertainties on their estimates, the evolutionary timeline of molecular clouds of M33 from Corbelli et al. (2017) and our analysis are in good agreement.

The time it takes for the star-forming regions to become exposed has also been measured using wavelengths other than $24 \mu \mathrm{m}$ as a tracer for the embedded star formation. Calzetti et al. (2015) have measured ages of young massive star clusters in the dwarf starburst galaxy NGC 5253 by applying SED modelling techniques on UVoptical-near-infrared Hubble Space Telescope photometry. While the star clusters have ages spanning from 1 to $15 \mathrm{Myr}$, the age estimate of one very heavily attenuated cluster with a clear near-infrared excess indicates that the duration of the heavily obscured phase of star formation is longer than (or similar to) $1 \mathrm{Myr}$ for this particular star-forming region. Whitmore et al. (2014) used free-free radio continuum emission to detect heavily obscured star-forming regions, and charaterized the evolutionary timeline from quiescent molecular clouds to exposed star-forming phase using age estimates from SED fitting of young stellar regions in the overlap region of the merging 
Antennae galaxies. The duration of the heavily obscured phase (referred to as Stage 2 in Whitmore et al. 2014) and the feedback time-scale (including the embedded phase; referred to as Stage 3 in Whitmore et al. 2014) are measured to be 0.1-1 and 1-3 Myr, somewhat shorter than the duration we measure with $24 \mu \mathrm{m}$, which are 1-4 and 2-7 Myr, respectively. We note that this difference could be because (i) the measurements are for galaxies undergoing a merger, unlike our sample; (ii) a different tracer is used to trace embedded star formation; and (iii) age estimates in highly extincted regions have considerable uncertainties (Hollyhead et al. 2015).

In conclusion, despite differences in methods, wavelengths, and galaxies used when constraining the evolutionary cycle of starforming regions, our results are in good agreement with the measured time-scales for the heavily obscured phase and feedback phase found in previous literature. The key step made in the present paper is to generalize these results to a sample of five galaxies (except M51), analysed homogeneously with a single analysis framework that is agnostic about which entities constitute a GMC or star-forming region.

\subsection{Effects of infrared emission not associated with local recent massive star formation}

$24 \mu \mathrm{m}$ emission is widely used as a tracer for embedded star formation, as it captures emission of massive stars that has been reprocessed by dust grains (see e.g. Calzetti et al. 2007; Kennicutt \& Evans 2012; Vutisalchavakul \& Evans 2013). However, one of the known issues with using $24 \mu \mathrm{m}$ emission to trace recent star formation is that the interstellar radiation field, late-type B stars (age of $\sim 100 \mathrm{Myr}$ ), and dust clumps heated by external radiation such as nearby star-forming regions also contribute to the emission at this wavelength (Calzetti et al. 2007; Murphy et al. 2011; Kennicutt \& Evans 2012; Leroy et al. 2012).

The difference in spatial distributions associated with each process generating $24 \mu \mathrm{m}$ emission allows us to separate the emission for recent star formation events from other sources. The $24 \mu \mathrm{m}$ emission originating from the interstellar radiation field has an extended morphology, because it originates from small dust grains in the diffuse interstellar medium (Draine \& Li 2007; Draine et al. 2007; Verley et al. 2009; Rahman et al. 2011; Leroy et al. 2012). Such diffuse emission, constituting on average of 55 per cent of the $24 \mu \mathrm{m}$ emission, is therefore expected to be removed during our filtering process.

On the other hand, the effect of $24 \mu \mathrm{m}$ emission associated with late-type B stars and starless dust clumps might not be filtered out because they are more inhomogeneously distributed, similarly to the emission from young star-forming regions. To estimate the effect of late-type B stars and externally illuminated dust clumps on our measurements, we make use of the far-infrared source catalogue of the LMC provided by Seale et al. (2014). In this catalogue, young stellar objects and dust clumps (that may or may not have deeply embedded forming stars) are identified, as well as sources not related to recent star formation such as asymptotic giant branch stars, planetary nebulae, and supernova remnants using literature catalogues (Seale et al. 2014 and references therein). In order to test whether the inclusion of $24 \mu \mathrm{m}$ emission from older stars and dust clumps could bias our results, we mask these sources and repeat our analysis of the LMC. When masking dust clumps, we mask all the probable candidates in Seale et al. (2014) as it is difficult to distinguish whether these clumps harbour deeply embedded stars or are heated by external radiation. We find that the older stars and dust clumps have a negligible effect on our results. In practice, older stars and dust clumps are not usually identified as SFR tracer peaks in our analysis due to their low brightness and small size, which does not satisfy the requirement of a minimum number of pixels to be identified as a peak in our method.

In conclusion, once the diffuse emission has been filtered, the $24 \mu \mathrm{m}$ maps mostly contain emission from young stars. Any potential bias due to the interstellar radiation field, late-type B stars, and dust clumps is negligible and our measurements of $t_{24} \mu \mathrm{m}$ provide an accurate characterization of the duration of (partially) embedded massive stars.

\section{CONCLUSION}

We present a characterization of the evolutionary timeline from molecular clouds to young stellar regions in six nearby galaxies by applying the statistical method developed by Kruijssen \& Longmore (2014) and Kruijssen et al. (2018) to CO and $24 \mu \mathrm{m}$ emission maps at cloud-scale $(20-100 \mathrm{pc})$ resolution. With this method, we measure the duration of the $24 \mu \mathrm{m}$ emission phase $\left(t_{24 \mu \mathrm{m}}\right)$, the duration of the feedback phase $\left(t_{\mathrm{fb}, 24 \mu \mathrm{m}}\right)$ during which massive star formation continues embedded in molecular clouds, the duration of the heavily obscured star formation phase with no associated $\mathrm{H} \alpha$ emission $\left(t_{\text {obscured }}\right)$, and the average distance between independent star-forming regions evolving from clouds to massive star formation $(\lambda)$. We also derive other physical quantities such as the feedback velocity $\left(v_{\mathrm{fb}}\right)$ and the integrated star formation efficiency per star formation event $\left(\epsilon_{\text {sf }}\right)$ from our measurements.

Across our sample of galaxies, we find that molecular clouds are quickly disrupted within 2-7 Myr after the onset of embedded massive star formation (traced by $24 \mu \mathrm{m}$ emission) by stellar feedback, supporting the fact that GMCs are dispersed within a cloud dynamical time-scale, as suggested by Elmegreen (2000) and Hartmann (2001). The measured feedback time-scale, which includes the duration of the massive star-forming phase, constitutes $17-47$ percent of the cloud lifetime of 10-30 Myr. The feedback time-scales are generally shorter than the time it takes for the first supernova to explode (4-20 Myr), when stochasticity of the initial mass function is taken into account (Chevance et al. 2020a), suggesting that early feedback mechanisms such as photoionization and stellar winds are mainly responsible for the dispersal of molecular clouds. Previous works have found similar duration of this phase using age estimates of star clusters in the Milky Way and some nearby galaxies (Lada \& Lada 2003; Whitmore et al. 2014; Corbelli et al. 2017). After the molecular gas is dispersed, the $24 \mu \mathrm{m}$ emission decays within 2-9 Myr. Our results further support the conclusion of earlier work that galaxies are composed of independent star-forming regions separated by $\sim 100-200 \mathrm{pc}$ (Kruijssen et al. 2019; Chevance et al. 2020c), which may correspond to the vertical gas disc scale height (see Kruijssen et al. 2019). These regions are undergoing an inefficient star-forming process with integrated cloud-scale star formation efficiencies $\left(\epsilon_{\mathrm{sf}}\right)$ of $0.7-6.8$ percent. The measured star formation efficiencies are consistent with previous measurements in these galaxies using other tracers to estimate the global SFR. We obtain feedback velocities $\left(v_{\mathrm{fb}}\right)$ of $8-30 \mathrm{~km} \mathrm{~s}^{-1}$, which is consistent with the observed expansion velocities of nearby H II regions (e.g. Murray \& Rahman 2010; McLeod et al. 2019, 2020; Barnes et al. 2020).

By combining our measurements with those using $\mathrm{H} \alpha$ as a tracer for exposed star-forming regions, we also measure the duration of the heavily obscured phase (detected with $\mathrm{CO}$ and $24 \mu \mathrm{m}$ but without $\mathrm{H} \alpha$ emission). Our results show that this period lasts for $3.0 \pm 0.9 \mathrm{Myr}$ (with a full range of 1.4-3.8 Myr across our sample of galaxies). We 
do not detect any significant correlation of the duration of this heavily obscured phase with galactic properties. This measured duration is in good agreement with values suggested by previous works using different wavelengths, methods, and galaxies.

Furthermore, we study the correlation of our measurements with galactic (or cloud-scale) properties, such as mass-weighted mean surface density of GMCs, metallicity, star formation efficiency, SFR surface density, and the feedback velocity. While we do not find statistically significant trends, the durations of the total and isolated $24 \mu \mathrm{m}$ emission phases $\left(t_{24} \mu \mathrm{m}\right.$ and $\left.t_{\text {iso, } 24 \mu \mathrm{m}}\right)$ may weakly decrease with increasing metallicity. We conjecture that this dependence results from winds of massive stars being stronger and more energetic at higher metallicities, which leads to a more effective dispersal of the clouds. No such trends with metallicity are observed for the feedback time-scale and the duration of the heavily obscured phase.

In order to gain a better understanding of the mechanisms driving the early feedback process, a systematic measurement of the embedded phase in a large number of galaxies in various environments is essential. Due to the limited resolution of Spitzer $24 \mu \mathrm{m}$ observations (6.4 $\mathrm{arcsec})$, we have been able to perform this analysis for only six nearby galaxies and accurately constrain the duration of the embedded phase in five of them. In the future, the MIRI imager aboard the James Webb Space Telescope, with a field of view of $1^{\prime} \times 2^{\prime}$, will reach an angular resolution of 0.7 arcsec. This will enable the application of the same method to galaxies located out to $25 \mathrm{Mpc}$, covering a much wider range of galaxy properties and morphologies, allowing us to explore how the feedback processes govern the evolution of molecular clouds during the early stages of star formation, as a function of the galactic environment.

\section{ACKNOWLEDGEMENTS}

We thank an anonymous referee for helpful comments that improved the quality of the manuscript. We thank Alexander Hygate for thankful suggestions and K. Herrmann for kindly sharing the $\mathrm{H} \alpha$ map of IC 342. JK, MC, and JMDK gratefully acknowledge funding from the German Research Foundation (DFG) through the DFG Sachbeihilfe (grant number KR4801/2-1). MC and JMDK gratefully acknowledge funding from the DFG through an Emmy Noether Grant (grant number KR4801/1-1). JMDK gratefully acknowledges funding from the European Research Council (ERC) under the European Union's Horizon 2020 research and innovation programme via the ERC Starting Grant MUSTANG (grant agreement number 714907). JMDK gratefully acknowledges funding from Sonderforschungsbereich SFB 881 (Project-ID 138713538) 'The Milky Way System' (subproject B2) of the DFG. FB and ATB would like to acknowledge funding from the European Research Council (ERC) under the European Union's Horizon 2020 research and innovation programme (grant agreement No.726384/Empire). SCOG and RSK acknowledge support from the Deutsche Forschungsgemeinschaft (DFG) via the Collaborative Research Center (SFB 881, Project-ID 138713538) 'The Milky Way System' (sub-projects A1, B1, B2, and B8) and from the Heidelberg cluster of excellence (EXC 2181 - 390900948) 'STRUCTURES: A unifying approach to emergent phenomena in the physical world, mathematics, and complex dat', funded by the German Excellence Strategy. They also thank for funding from the European Research Council in the ERC Synergy Grant 'ECOGAL Understanding our Galactic ecosystem: From the disc of the Milky Way to the formation sites of stars and planets' (project ID 855130). KK gratefully acknowledges funding from the German Research Foundation (DFG) in the form of an Emmy Noether Research Group (grant number KR4598/2-1, PI Kreckel). The work of AKL and JS is partially supported by the National Science Foundation (NSF) under grant nos. 1615105, 1615109, and 1653300. MQ acknowledges support from the research project PID2019-106027GA-C44 from the Spanish Ministerio de Ciencia e Innovación. ES and TGW acknowledge funding from the European Research Council (ERC) under the European Union's Horizon 2020 research and innovation programme (grant agreement no. 694343). This work was carried out as part of the PHANGS collaboration. This paper makes use of the following ALMA data: ADS/JAO.ALMA \#2013.1.00351.S, ADS/JAO.ALMA \#2015.1.00258.S. ALMA is a partnership of ESO (representing its member states), NSF (USA) and NINS (Japan), together with NRC (Canada), NSC and ASIAA (Taiwan), and KASI (Republic of Korea), in cooperation with the Republic of Chile. The Joint ALMA Observatory is operated by ESO, AUI/NRAO, and NAOJ. The National Radio Astronomy Observatory is a facility of the National Science Foundation operated under cooperative agreement by Associated Universities, Inc. This work makes use of the PdBI Arcsecond Whirlpool Survey (Pety et al. (2013); Schinnerer et al. (2013)). The authors thank IRAM for making the data products of IC 342 (Schruba et al. 2021a), M31 (Schruba et al. 2021b), and M33 CO Large Program (Gratier et al. 2010; Druard et al. 2014) available. IRAM is supported by INSU/CNRS (France), MPG (Germany), and IGN (Spain). We thank the IRAM staff for their assistance with the observations.

\section{DATA AVAILABILITY}

The data underlying this article will be shared on reasonable request to the corresponding author.

\section{REFERENCES}

Alves J. et al., 2020, Nature, 578, 237

Aniano G., Draine B. T., Gordon K. D., Sandstrom K., 2011, PASP, 123, 1218

Asplund M., Grevesse N., Sauval A. J., Scott P., 2009, ARA\&A, 47, 481

Azimlu M., Marciniak R., Barmby P., 2011, AJ, 142, 139

Backus C., Velusamy T., Thompson T., Arballo J., 2005, Astronomical Data Analysis Software and Systems XIV, 347, Hires: Super-resolution for the Spitzer Space Telescope. p. 61

Barnes A. T., Longmore S. N., Dale J. E., Krumholz M. R., Kruijssen J. M. D., Bigiel F., 2020, MNRAS, 498, 4906

Bigiel F., Leroy A., Walter F., Brinks E., de Blok W. J. G., Madore B. Thornley M. D., 2008, AJ, 136, 2846

Blitz L., Fukui Y., Kawamura A., Leroy A., Mizuno N., Rosolowsky E., 2007, in Reipurth B., Jewitt D., Keil K., eds, Protostars and Planets V. p. 81

Böker T., van der Marel R. P., Vacca W. D., 1999, AJ, 118, 831

Bolatto A. D., Wolfire M., Leroy A. K., 2013, ARA\&A, 51, 207

Bresolin F., 2011, ApJ, 730, 129

Caldú-Primo A., Schruba A., 2016, AJ, 151, 34

Calzetti D. et al., 2005, ApJ, 633, 871

Calzetti D. et al., 2007, ApJ, 666, 870

Calzetti D. et al., 2015, ApJ, 811, 75

Cardelli J. A., Clayton G. C., Mathis J. S., 1989, ApJ, 345, 245

Chevance M. et al., 2020a, preprint (arXiv:2010.13788)

Chevance M. et al., 2020b, Space Sci. Rev., 216, 50

Chevance M. et al., 2020c, MNRAS, 493, 2872

Colombo D. et al., 2014, ApJ, 784, 4

Corbelli E. et al., 2017, A\&A, 601, A146

Corbelli E., Lorenzoni S., Walterbos R., Braun R., Thilker D., 2010, A\&A, 511, A89

da Silva R. L., Fumagalli M., Krumholz M., 2012, ApJ, 745, 145

da Silva R. L., Fumagalli M., Krumholz M. R., 2014, MNRAS, 444, 3275

Dalcanton J. J. et al., 2009, ApJS, 183, 67

Dalcanton J. J. et al., 2012, ApJS, 200, 18 
Dale D. A. et al., 2007, ApJ, 655, 863

Dale D. A. et al., 2009, ApJ, 703, 517

Dale J. E., 2015, New A Rev., 68, 1

Draine B. T. et al., 2007, ApJ, 663, 866

Draine B. T., Li A., 2007, ApJ, 657, 810

Druard C. et al., 2014, A\&A, 567, A118

Dumas G., Schinnerer E., Tabatabaei F. S., Beck R., Velusamy T., Murphy E., 2011, AJ, 141, 41

Efremov Y. N., Elmegreen B. G., 1998, MNRAS, 299, 588

Elmegreen B. G., 2000, ApJ, 530, 277

Elmegreen B. G., Elmegreen D. M., 2019, ApJS, 245, 14

Elmegreen B. G., Elmegreen D. M., 2020, ApJ, 895, 71

Engargiola G., Plambeck R. L., Rosolowsky E., Blitz L., 2003, ApJS, 149, 343

Eufrasio R. T. et al., 2017, ApJ, 851, 10

Faesi C. M., Lada C. J., Forbrich J., Menten K. M., Bouy H., 2014, ApJ, 789, 81

Feldmann R., Gnedin N. Y., Kravtsov A. V., 2011, ApJ, 732, 115

Fitzpatrick E. L., 1999, PASP, 111, 63

Fitzpatrick E. L., Massa D., 2007, ApJ, 663, 320

Ford G. P. et al., 2013, ApJ, 769, 55

Fukui Y. et al., 2008, ApJS, 178, 56

Gaia Collaboration, 2018, A\&A, 616, A1

Gaustad J. E., McCullough P. R., Rosing W., Van Buren D., 2001, PASP, 113, 1326

Gieren W. et al., 2013, ApJ, 773, 69

Gordon K. D. et al., 2006, ApJ, 638, L87

Grasha K. et al., 2018, MNRAS, 481, 1016

Grasha K. et al., 2019, MNRAS, 483, 4707

Gratier P. et al., 2010, A\&A, 522, A3

Greenawalt B. E., 1998, PhD thesis, New Mexico State University

Harris J., Zaritsky D., 2009, AJ, 138, 1243

Hartmann L., 2001, AJ, 121, 1030

Haydon D. T., Fujimoto Y., Chevance M., Kruijssen J. M. D., Krumholz M. R., Longmore S. N., 2020a, MNRAS, 497, 5076

Haydon D. T., Kruijssen J. M. D., Chevance M., Hygate A. P. S., Krumholz M. R., Schruba A., Longmore S. N., 2020b, MNRAS, 498, 235

Henshaw J. D. et al., 2020, Nat. Astron., 4, 1064

Herrera C. N. et al., 2020, A\&A, 634, A121

Heyer M., Dame T. M., 2015, ARA\&A, 53, 583

Hollyhead K., Bastian N., Adamo A., Silva-Villa E., Dale J., Ryon J. E., Gazak Z., 2015, MNRAS, 449, 1106

Hoopes C. G., Walterbos R. A. M., 2000, ApJ, 541, 597

Hygate A. P. S., 2020, PhD thesis, Ruperto-Carola-University of Heidelberg

Hygate A. P. S., Kruijssen J. M. D., Chevance M., Schruba A., Haydon D. T., Longmore S. N., 2019, MNRAS, 488, 2800

Jacobs B. A., Rizzi L., Tully R. B., Shaya E. J., Makarov D. I., Makarova L., 2009, AJ, 138, 332

Jameson K. E. et al., 2016, ApJ, 825, 12

Kang X., Chang R., Yin J., Hou J., Zhang F., Zhang Y., Han Z., 2012, MNRAS, 426, 1455

Kang X., Zhang F., Chang R., Wang L., Cheng L., 2016, A\&A, 585, A20

Kawamura A. et al., 2009, ApJS, 184, 1

Kelly B. C., 2007, ApJ, 665, 1489

Kennicutt Robert C. J. et al., 2003, PASP, 115, 928

Kennicutt Robert C. J. et al., 2007, ApJ, 671, 333

Kennicutt Robert C. J., 1998a, ARA\&A, 36, 189

Kennicutt Robert C. J., 1998b, ApJ, 498, 541

Kennicutt R. C., Evans N. J., 2012, ARA\&A, 50, 531

Kim S., Staveley-Smith L., Dopita M. A., Freeman K. C., Sault R. J., Kesteven M. J., McConnell D., 1998, ApJ, 503, 674

Kim J.-G., Kim W.-T., Ostriker E. C., 2018, ApJ, 859, 68

Kim J.-G., Ostriker E. C., Filippova N., 2020, preprint (arXiv:2011.07772)

Koch E. W. et al., 2018, MNRAS, 479, 2505

Koepferl C. M., Robitaille T. P., Morales E. F. E., Johnston K. G., 2015, ApJ, 799, 53

Kreckel K. et al., 2018, ApJ, 863, L21

Kruijssen J. M. D. et al., 2019, Nature, 569, 519
Kruijssen J. M. D., Longmore S. N., 2014, MNRAS, 439, 3239

Kruijssen J. M. D., Schruba A., Hygate A. e. P. S., Hu C.-Y., Haydon D. T., Longmore S. N., 2018, MNRAS, 479, 1866

Krumholz M. R., 2014, Phys. Rep., 539, 49

Krumholz M. R., Fumagalli M., da Silva R. L., Rendahl T., Parra J., 2015, MNRAS, 452, 1447

Lada C. J., Lada E. A., 2003, ARA\&A, 41, 57

Leitherer C. et al., 1999, ApJS, 123, 3

Leitherer C., Ekström S., Meynet G., Schaerer D., Agienko K. B., Levesque E. M., 2014, ApJS, 212, 14

Leroy A. K. et al., 2012, AJ, 144, 3

Leroy A. K. et al., 2013, AJ, 146, 19

Leroy A. K. et al., 2017, ApJ, 846, 71

Leroy A. K. et al., 2019, ApJS, 244, 24

Lewis A. R. et al., 2015, ApJ, 805, 183

Lockman F. J., 1989, ApJS, 71, 469

Lucas W. E., Bonnell I. A., Dale J. E., 2020, MNRAS, 493, 4700

Maeder A., 1992, A\&A, 264, 105

Makarov D., Prugniel P., Terekhova N., Courtois H., Vauglin I., 2014, A\&A, 570, A13

Martin C. L., 1997, ApJ, 491, 561

Massey P., Olsen K. A. G., Hodge P. W., Strong S. B., Jacoby G. H., Schlingman W., Smith R. C., 2006, AJ, 131, 2478

McLeod A. F. et al., 2020, ApJ, 891, 25

McLeod A. F., Dale J. E., Evans C. J., Ginsburg A., Kruijssen J. M. D., Pellegrini E. W., Ramsay S. K., Testi L., 2019, MNRAS, 486, 5263

Meidt S. E., Rand R. J., Merrifield M. R., 2009, ApJ, 702, 277

Meixner M. et al., 2006, AJ, 132, 2268

Miura R. E. et al., 2012, ApJ, 761, 37

Murphy E. J., Chary R. R., Dickinson M., Pope A., Frayer D. T., Lin L., 2011, ApJ, 732, 126

Murray N., Rahman M., 2010, ApJ, 709, 424

Nazé Y., Chu Y.-H., Points S. D., Danforth C. W., Rosado M., Chen C. H. R., 2001, AJ, 122, 921

Nazé Y., Chu Y.-H., Guerrero M. A., Oey M. S., Gruendl R. A., Smith R. C., 2002, AJ, 124, 3325

Nersesian A. et al., 2020, A\&A, 643, A90

Nieten C., Neininger N., Guélin M., Ungerechts H., Lucas R., Berkhuijsen E. M., Beck R., Wielebinski R., 2006, A\&A, 453, 459

Onodera S. et al., 2010, ApJ, 722, L127

Patra N. N., 2020, A\&A, 638, A66

Paturel G., Theureau G., Bottinelli L., Gouguenheim L., Coudreau-Durand N., Hallet N., Petit C., 2003a, A\&A, 412, 57

Paturel G., Theureau G., Bottinelli L., Gouguenheim L., Coudreau-Durand N., Hallet N., Petit C., 2003b, A\&A, 412, 57

Pety J. et al., 2013, ApJ, 779, 43

Pietrzyński G. et al., 2019, Nature, 567, 200

Pilyugin L. S., 2001, A\&A, 369, 594

Pilyugin L. S., Grebel E. K., Mattsson L., 2012, MNRAS, 424, 2316

Pilyugin L. S., Grebel E. K., Zinchenko I. A., Kniazev A. Y., 2014, AJ, 148, 134

Prescott M. K. M. et al., 2007, ApJ, 668, 182

Querejeta M. et al., 2019, A\&A, 625, A19

Rahman N. et al., 2011, ApJ, 730, 72

Rémy-Ruyer A. et al., 2014, A\&A, 563, A31

Rieke G. H. et al., 2004, ApJS, 154, 25

Schinnerer E. et al., 2013, ApJ, 779, 42

Schinnerer E. et al., 2019, ApJ, 887, 49

Schlafly E. F., Finkbeiner D. P., 2011, ApJ, 737, 103

Schlegel D. J., Finkbeiner D. P., Davis M., 1998, ApJ, 500, 525

Schmidt M., 1959, ApJ, 129, 243

Schruba A. et al., 2021a, ApJ, to be submitted

Schruba A., Leroy A. K., Walter F., Sandstrom K., Rosolowsky E., 2010, ApJ, 722, 1699

Schruba A., Kruijssen J. M. D., Leroy A. K., 2019, ApJ, 883, 2

Schruba A., Leroy A. K., Bolatto A. D., Dalcanton J. J., Sandstrom K. M., Scoville N., Walter F., Weisz D. R., 2021b, ApJ, to be submitted

Schuster K. F. et al., 2004, A\&A, 423, 1171 
Scoville N. Z., Thakkar D., Carlstrom J. E., Sargent A. I., 1993, ApJ, 404, L59

Seale J. P. et al., 2014, AJ, 148, 124

Sharma S., Corbelli E., Giovanardi C., Hunt L. K., Palla F., 2011, A\&A, 534, A96

Sick J., Courteau S., Cuillandre J.-C., Dalcanton J., de Jong R., McDonald M., Simard D., Tully R. B., 2015, in Cappellari M., Courteau S., eds, Vol. 311, Galaxy Masses as Constraints of Formation Models, p. 82

Silk J., 1997, ApJ, 481, 703

Skibba R. A. et al., 2012, ApJ, 761, 42

Staveley-Smith L., Kim S., Calabretta M. R., Haynes R. F., Kesteven M. J., 2003, MNRAS, 339, 87

Toribio San Cipriano L., García-Rojas J., Esteban C., Bresolin F., Peimbert M., 2016, MNRAS, 458, 1866

Toribio San Cipriano L., Domínguez-Guzmán G., Esteban C., García-Rojas J., Mesa-Delgado A., Bresolin F., Rodríguez M., Simón-Díaz S., 2017, MNRAS, 467, 3759

Utomo D. et al., 2018, ApJ, 861, L18

Verley S., Corbelli E., Giovanardi C., Hunt L. K., 2009, A\&A, 493, 453

Viaene S. et al., 2017, A\&A, 599, A64

Vutisalchavakul N., Evans Neal J. I., 2013, ApJ, 765, 129

Ward J. L., Chevance M., Kruijssen J. M. D., Kim J. J., Hygate A. P. S., Schruba A., Longmore S. N., 2020a, MNRAS, to be submitted

Ward J. L., Chevance M., Kruijssen J. M. D., Hygate A. P. S., Schruba A., Longmore S. N., 2020b, MNRAS, 497, 2286
Westmeier T., Braun R., Koribalski B. S., 2011, MNRAS, 410, 2217

Whitmore B. C. et al., 2014, ApJ, 795, 156

Williams B. F. et al., 2017, ApJ, 846, 145

Williams T. G., Gear W. K., Smith M. W. L., 2018, MNRAS, 479, 297

Williams T. G., Baes M., De Looze I., Relaño M., Smith M. W. L., Verstocken S., Viaene S., 2019, MNRAS, 487, 2753

Williams J. P., de Geus E. J., Blitz L., 1994, ApJ, 428, 693

Wong T. et al., 2011, ApJS, 197, 16

Wong T. et al., 2017, ApJ, 850, 139

Wood K., Hill A. S., Joung M. R., Mac Low M.-M., Benjamin R. A., Haffner L. M., Reynolds R. J., Madsen G. J., 2010, ApJ, 721, 1397

Wu P.-F., Tully R. B., Rizzi L., Dolphin A. E., Jacobs B. A., Karachentsev I. D., 2014, AJ, 148, 7

Yim K., Wong T., Xue R., Rand R. J., Rosolowsky E., van der Hulst J. M., Benjamin R., Murphy E. J., 2014, AJ, 148, 127

Yim K., Wong T., Rand R. J., Schinnerer E., 2020, MNRAS, 494, 4558

Zabel N. et al., 2020, MNRAS, 496, 2155

Zurita A., Bresolin F., 2012, MNRAS, 427, 1463

\section{APPENDIX A: INDIVIDUAL IMAGES OF EMISSION MAPS USED IN OUR ANALYSIS}

In Figs $\mathrm{A} 1$ and A2, we present the $\mathrm{CO}$ and $24 \mu \mathrm{m}$ emission maps used in our analysis to trace molecular gas and young stellar regions, respectively. 

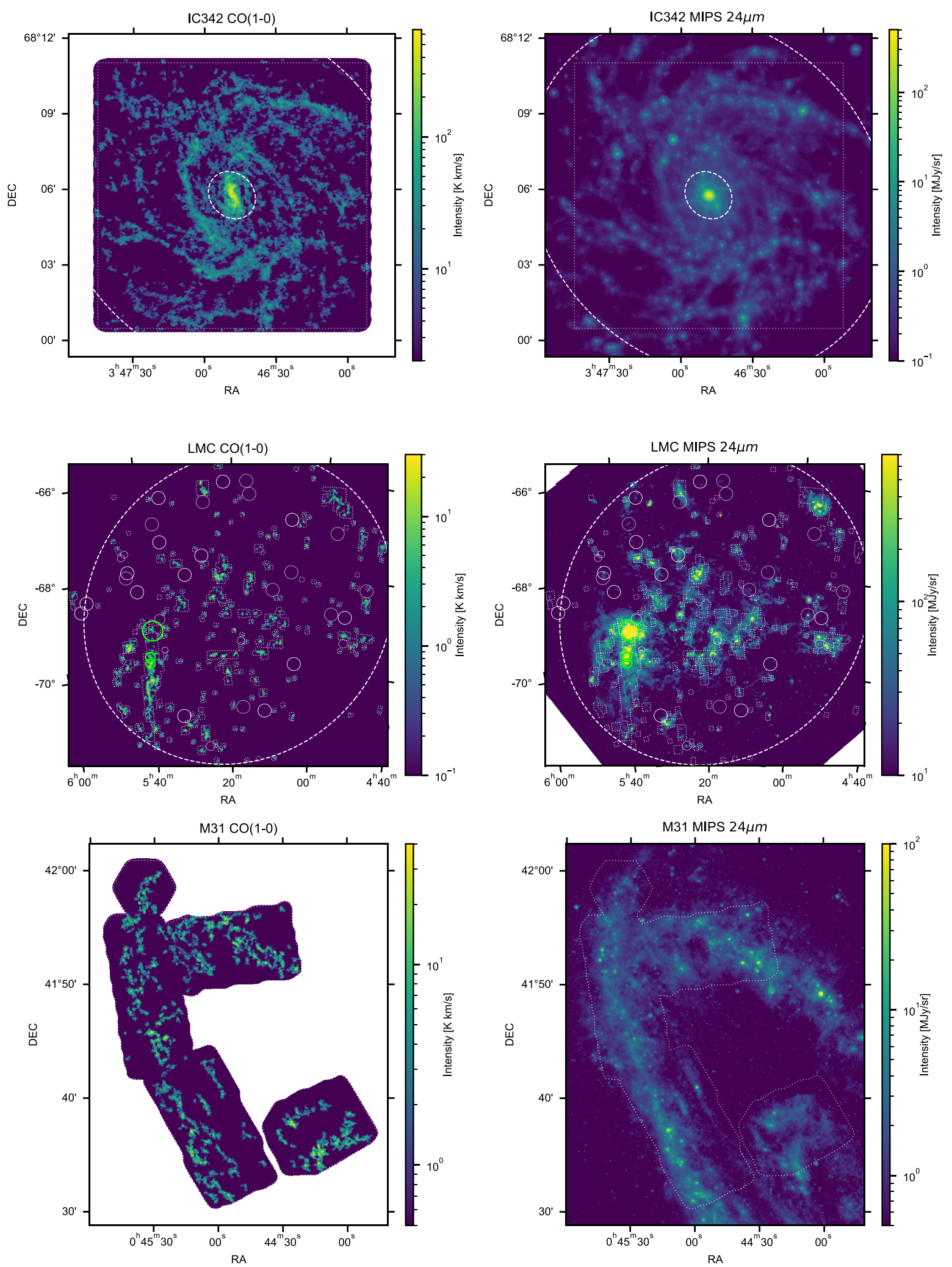

Figure A1. Maps of $\mathrm{CO}(1-0)$ emission (left-hand panels) and $24 \mu \mathrm{m}$ emission (right-hand panels) for the IC 342, LMC, and M31. The ranges of galactic radii included in our analysis are indicated by white dashed ellipses. The white dotted line shows the coverage of the CO observations. In the LMC, regions where molecular gas exists but was not targeted by the MAGMA survey are masked (white solid circles). The masked bright star-forming regions are shown by green circles. We also mask foreground stars, background galaxies, and map artefacts (purple circles). 

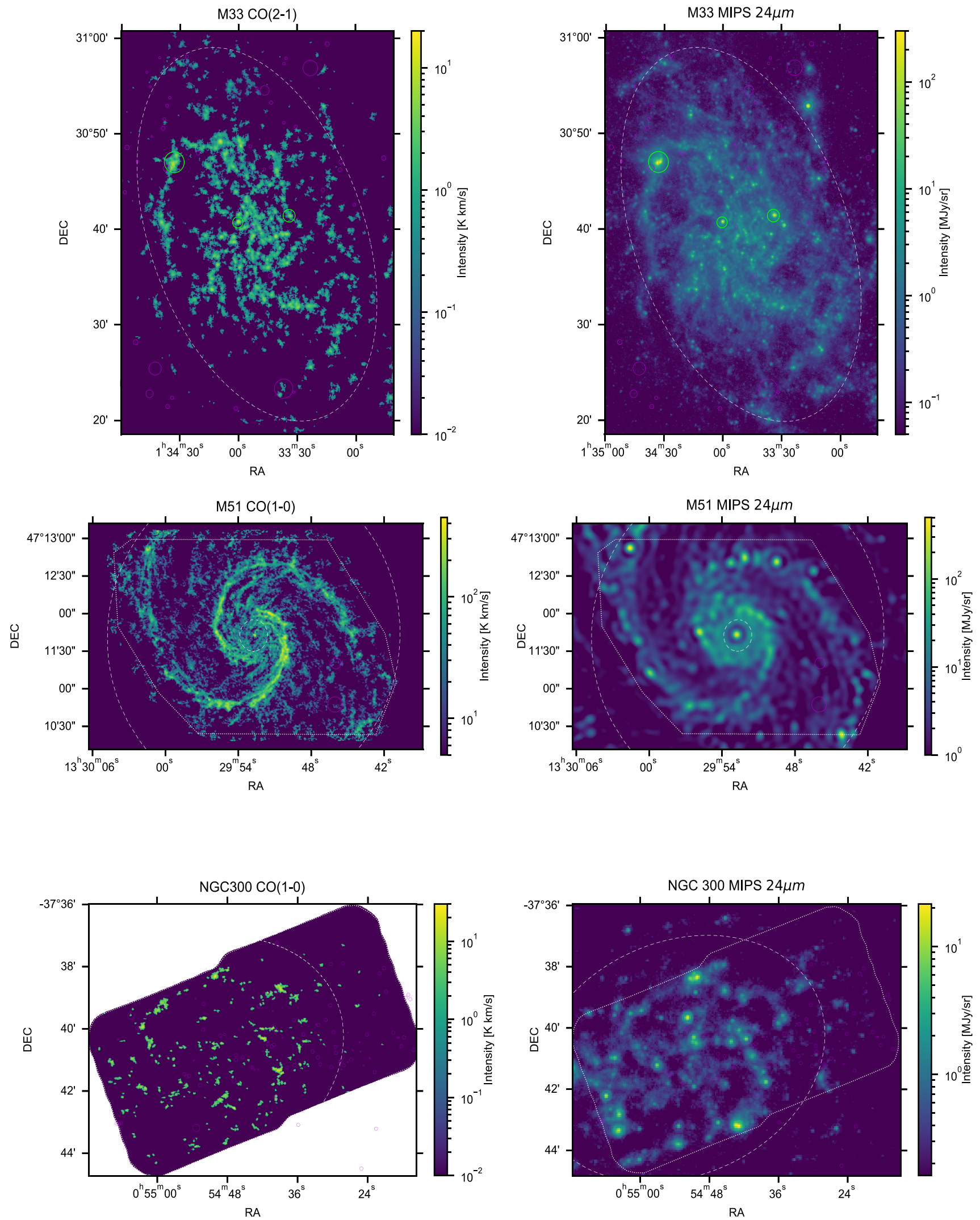

Figure A2. Same as Fig. A1, but here for M33, M51, and NGC 300. Integrated intensity maps of CO(1-0) are shown for M51 and NGC 300, while CO(2-1) is shown for M33. 


\section{APPENDIX B: MOLECULAR CLOUD LIFETIMES IN IC 342 AND M31}

In order to derive absolute durations of the different phases of cloud evolution and star formation (see Section 3), we have used the cloud lifetime $\left(t_{\mathrm{CO}}\right)$ as the reference time-scale in our analysis (see Table 2). For four out of six galaxies in our sample, the cloud lifetime has been constrained in previous works using $\mathrm{H} \alpha$ as a tracer for exposed star-forming regions. Here, we describe the characterization of the cloud lifecycle of IC 342 and M31, by applying the same method as described in Section 3 using $\mathrm{CO}$ and $\mathrm{H} \alpha$ emission maps to trace molecular gas and young stellar regions, respectively.

Fig. B1 shows the $\mathrm{H} \alpha$ emission maps of IC 342 and M31 used to trace young massive star-forming regions. A summary of the observational data is presented in Section 2.1. Table B1 lists the adopted main input parameters specific to the analysis using $\mathrm{CO}$
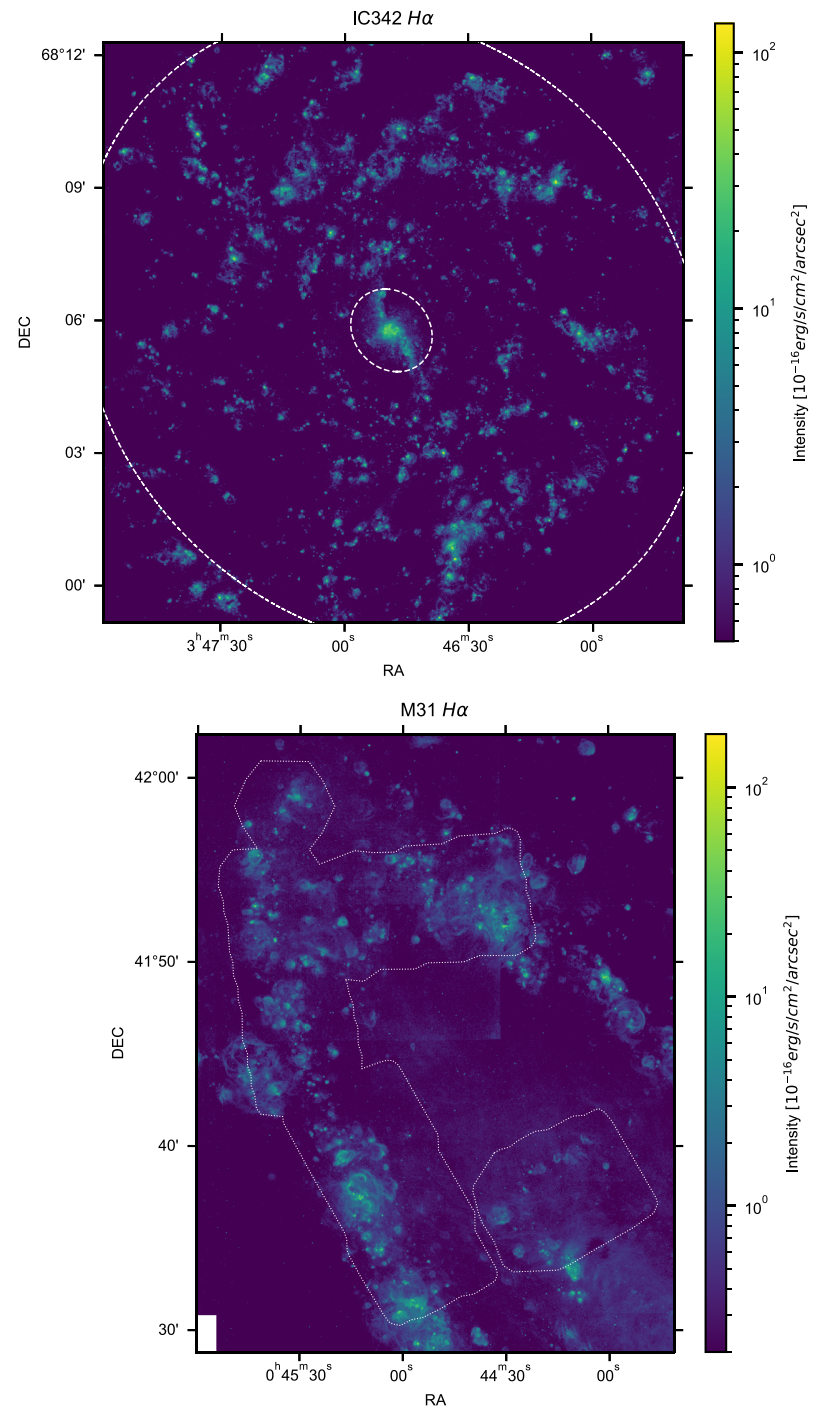

Figure B1. Same as Fig. A2, for $\mathrm{H} \alpha$ emission maps of IC 342 and M31.
Table B1. Main input parameters of the analysis using $\mathrm{H} \alpha$ as an SFR tracer for IC 342 and M31. For other input parameters, we use the default values listed in table 2 of Kruijssen et al. (2018).

\begin{tabular}{lcc}
\hline Quantity & IC 342 & M31 \\
\hline$l_{\text {ap, min }}(\mathrm{pc})$ & 65 & 45 \\
$l_{\text {ap, max }}(\mathrm{pc})$ & 3000 & 4000 \\
$N_{\text {ap }}$ & 15 & 15 \\
$N_{\text {pix,min }}$ & 10 & 20 \\
$\Delta \log _{10} \mathcal{F}_{\mathrm{CO}}$ & 1.1 & 1.2 \\
$\delta \log _{10} \mathcal{F}_{\mathrm{CO}}$ & 0.05 & 0.05 \\
$\Delta \log _{10} \mathcal{F}_{\mathrm{H} \alpha}$ & 2.8 & 2.0 \\
$\delta \log _{10} \mathcal{F}_{\mathrm{H} \alpha}$ & 0.05 & 0.1 \\
$t_{\text {ref }}(\mathrm{Myr})$ & 4.25 & 4.42 \\
$t_{\text {ref, errmin }}(\mathrm{Myr})$ & 0.15 & 0.18 \\
$t_{\text {ref, errmax }}(\mathrm{Myr})$ & 0.15 & 0.19 \\
$n_{\lambda}$ & 12 & 10 \\
\hline
\end{tabular}

and $\mathrm{H} \alpha$ as molecular gas and SFR tracers, respectively. Other input parameters are listed in Tables 1 and 2 . We set the minimum aperture size $\left(l_{\mathrm{ap}, \min }\right)$ to match the $\mathrm{CO}$ map resolution as it is coarser compared to the $\mathrm{H} \alpha$ map resolution. The reference time-scales $\left(t_{\text {ref }}\right)$ for the exposed young stellar phase (duration of the isolated $\mathrm{H} \alpha$ emission phase) are adopted from Haydon et al. (2020b). The duration does not include the feedback time-scale, so the total duration of the $\mathrm{H} \alpha$ emission phase $\left(t_{\mathrm{H} \alpha}\right)$ equals $t_{\mathrm{ref}}+t_{\mathrm{fb}, \mathrm{H} \alpha}$.

In Fig. B2, we show the measured deviations of the enclosed gas-to-SFR flux ratios in apertures centred on $\mathrm{CO}$ and $\mathrm{H} \alpha$ peaks, compared to the galactic average, together with our best-fitting model. Table 3 lists the constrained quantities for the best-fitting model. We have verified the accuracy of these measurements in Section 5.1. As seen in our results using $24 \mu \mathrm{m}$ as an SFR tracer, we find a spatial de-correlation between gas and SFR tracer emission peaks. We measure cloud lifetimes of $20.0_{-2.3}^{+2.0} \mathrm{Myr}$ for IC 342 and $14.0_{-1.9}^{+2.1}$ Myr for M31. The measured cloud lifetimes are within the range of our previous measurements of other galaxies where GMCs are found to live for 10-30 Myr (Kruijssen et al. 2019; Chevance et al. 2020c; Hygate 2020; Ward et al. 2020a; Zabel et al. 2020). We suspect that the difference between the two cloud lifetimes are related to the different environments in which the molecular clouds are located, as recently suggested by Chevance et al. (2020c). In this case, the cloud evolution in IC 342, which has a high molecular gas surface density $\left(9.55 \mathrm{M}_{\odot} \mathrm{pc}^{-2}\right)$, is likely to be governed by galactic dynamical processes, whereas internal dynamics such as free-fall and crossing times are the determinant factor for cloud evolution in low molecular gas surface density environments such as $\mathrm{M} 31\left(\sim 1 \mathrm{M}_{\odot} \mathrm{pc}^{-2}\right)$. The duration over which $\mathrm{CO}$ and $\mathrm{H} \alpha$ emission overlap is short $\left(2.2_{-0.5}^{+0.4} \mathrm{Myr}\right.$ in IC 342 and $1.1_{-0.2}^{+0.3}$ Myr in M31), as seen in previous measurements of other galaxies (Chevance et al. 2020a). These short feedback time-scales indicate that molecular clouds are destroyed shortly after the starforming region becomes exposed, making them visible in $\mathrm{H} \alpha$. Finally, we find that independent star-forming regions are separated by $120_{-10}^{+10} \mathrm{pc}$ in IC 342 and $181_{-19}^{+28} \mathrm{pc}$ in M31, comparable to our measurements with $24 \mu \mathrm{m}$ as an SFR tracer, as well as our previous findings with $\mathrm{H} \alpha$ for different galaxies. 

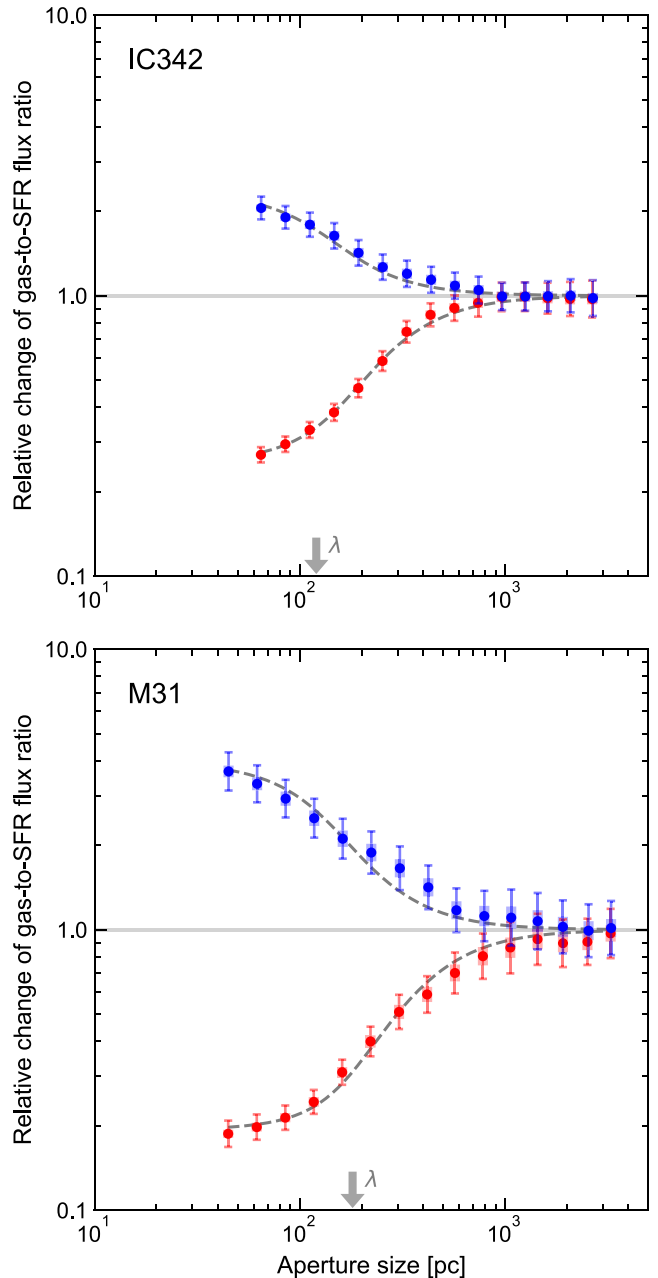

Figure B2. Relative change of the gas-to-SFR (CO-to-H $\alpha)$ flux ratio compared to the galactic average as a function of size of apertures placed on $\mathrm{CO}$ (blue) and $\mathrm{H} \alpha$ (red) emission peaks. The error bars indicate $1 \sigma$ uncertainty on each individual data point whereas the shaded area is an effective $1 \sigma$ uncertainty taking into account the covariance between data points. Our best-fitting model is shown as dashed line and the solid horizontal line indicates the galactic average. The measured region separation length $(\lambda)$ is indicated in each panel and other constrained best-fitting parameters $\left(t_{\mathrm{CO}}\right.$ and $\left.t_{\mathrm{fb}, \mathrm{H} \alpha}\right)$ are listed in Table 3 .
${ }^{1}$ Astronomisches Rechen-Institut, Zentrum für Astronomie der Universität Heidelberg, Mönchhofstraße 12-14, D-69120 Heidelberg, Germany

${ }^{2}$ Max-Planck Institut für Extraterrestrische Physik, Giessenbachstraße 1, D85748 Garching, Germany

${ }^{3}$ Center for Astrophysics and Space Sciences, Department of Physics, University of California, San Diego, 9500 Gilman Drive, La Jolla, CA 92093 , USA

${ }^{4}$ Argelander-Institut für Astronomie, Universität Bonn, Auf dem Hügel 71, D-53121 Bonn, Germany

${ }^{5}$ The Observatories of the Carnegie Institution for Science, 813 Santa Barbara Street, Pasadena, CA 91101, USA

${ }^{6}$ Departamento de Astronomía, Universidad de Chile, Casilla 36-D, Santiago, Chile

${ }^{7}$ Aix Marseille Univ, CNRS, CNES, LAM (Laboratoire d'Astrophysique de Marseille), Marseille, France

${ }^{8}$ Department of Physics \& Astronomy, University of Wyoming, Laramie, WY 8207, USA

${ }^{9}$ Department of Astronomy, University of Massachusetts - Amherst, $710 \mathrm{~N}$. Pleasant St., Amherst, MA 01003, USA

${ }^{10}$ Institüt für Theoretische Astrophysik, Zentrum für Astronomie der Universität Heidelberg, Albert-Ueberle-Strasse 2, D-69120 Heidelberg, Germany

${ }^{11}$ Research School of Astronomy and Astrophysics, Australian National University, Canberra, ACT 2611, Australia

${ }^{12}$ International Centre for Radio Astronomy Research, University of Western Australia, 7 Fairway, Crawley, WA 6009, Australia

${ }^{13}$ IRAM, 300 rue de la Piscine, F-38406 Saint Martin d'Hères, France

${ }^{14}$ Universität Heidelberg, Interdisziplinäres Zentrum für Wissenschaftliches Rechnen, Im Neuenheimer Feld 205, D-69120 Heidelberg, Germany

${ }^{15}$ Caltech/IPAC MC 314-6 (Keith Spalding Building) 1200 E California Blvd Pasadena, CA 91125, USA

${ }^{16}$ Department of Astronomy, The Ohio State University, 140 West 18th Ave, Columbus, $\mathrm{OH} 43210$, USA

${ }^{17}$ Sorbonne Université, Observatoire de Paris, Université PSL, CNRS, LERMA, F-75005 Paris, France

${ }^{18}$ Observatorio Astronómico Nacional (IGN), C/Alfonso XII 3, Madrid E28014, Spain

${ }^{19}$ Max Planck Institut für Astronomie, Königstuhl 17, D-69117 Heidelberg, Germany

This paper has been typeset from a $\mathrm{T}_{\mathrm{E}} \mathrm{X} / \mathrm{L} \mathrm{T}_{\mathrm{E}} \mathrm{X}$ file prepared by the author. 